\title{
AMRNodeElliptic User Guide: on irregular problem domains
}

\author{
Peter McCorquodale
}

April 15, 2003 


\section{Disclaimer}

This document was prepared as an account of work sponsored by the United States Government. While this document is believed to contain correct information, neither the United States Government nor any agency thereof, nor The Regents of the University of California, nor any of their employees, makes any warranty, express or implied, or assumes any legal responsibility for the accuracy, completeness, or usefulness of any information, apparatus, product, or process disclosed, or represents that its use would not infringe privately owned rights. Reference herein to any specific commercial product, process, or service by its trade name, trademark, manufacturer, or otherwise, does not necessarily constitute or imply its endorsement, recommendation, or favoring by the United States Government or any agency thereof, or The Regents of the University of California, The views and opinion authors expressed herein do not necessarily state or reflect those of the United States Government or any agency thereof, or The Regents of the University of California. 


\section{Contents}

1 Introduction 4

1.1 Constructing a makefile with AMRNodeElliptic . . . . . . . . . . . 4

2 Subroutines Needed for Non-Rectangular Domains 6

2.1 The reachablenodes subroutine . . . . . . . . . . . . . 6

2.2 The nodalcoefficients subroutine . . . . . . . . . . . 7

3 Node-Centered Data 9

3.1 The class NodeFArrayBox . . . . . . . . . . . . . . . . . . . 9

3.2 Notes on LevelData $<$ NodeFArrayBox $>$ : : copyTo . . . . . . . . . . . 11

3.3 Node-centered norms . . . . . . . . . . . . . . . . . . . 12

3.3.1 Norms of valid data on a single level . . . . . . . . . . . 12

3.3.2 Norms of data on multiple levels . . . . . . . . . . . . . 13

3.4 Dot product for node-centered data . . . . . . . . . . . . . . 14

3.5 Functions for interior and exterior boundary nodes . . . . . . . . 15

3.5.1 interiorBoundaryNodes functions . . . . . . . . . . 15

3.5 .2 exteriorBoundaryNodes function . . . . . . . . . . . 18

3.5 .3 copyInteriorNodes function . . . . . . . . . . . . . . . . 19

3.5.4 zeroBoundaryNodes function . . . . . . . . . . . . . . . . . 19

4 Interface for AMRNodeElliptic Solver 22

4.1 The class AMRNodeSolver . . . . . . . . . . . . . . . . . . . 22

4.1 .1 Internal class AMRNodeLevelMG . . . . . . . . . . . 27

4.1 .2 Internal class NodeLevelMG . . . . . . . . . . . . . . . . . 29

4.2 The class LevelNodeSolver . . . . . . . . . . . . . . . . . . . 31

4.3 The NodeMaskLevelOp interface . . . . . . . . . . . . . . . 34

4.3.1 The NodeMaskBaseBottomSmoother interface . . . . . . . . 38

4.3.2 The class NodeMaskPoissonOp . . . . . . . . . . . . . . . . . 39

4.4 The class AMRNodeSolverAlt . . . . . . . . . . . . . . . . . . . . . . 39

$5 \mathrm{C}++$ Classes for Two-Level Operators 42

5.1 The class NodeMaskAverage . . . . . . . . . . . . . . . . . . . . . 42

5.2 The class NodeMGInterp . . . . . . . . . . . . . . . . 43 
5.3 The class NodeQuadCFInterp . . . . . . . . . . . . . . . . . . . . . . 44

5.4 The class NodeCFIVS . . . . . . . . . . . . . . . . . . . . . . . 46

6 External Boundary Conditions $\quad 48$

6.1 The DomainNodeBC class . . . . . . . . . . . . . . . . . . . . . . . . . 48

6.2 The FaceNodeBC interface . . . . . . . . . . . . . . . . . . . . . . . . 49 


\section{Chapter 1}

\section{Introduction}

This document describes an extension to the Chombo package [ $\mathrm{CGL}^{+} 00$ ] for solving elliptic equations using adaptive mesh refinement on multiple levels with node-centered data on non-rectangular domains. See the AMRNodeElliptic design document [McC02] for a description of the algorithms used.

Chapter 2 describes the subroutines that the user must supply to describe the geometry and the operator. Chapter 3 describes the class NodeFArrayBox and functions that use this class to manipulate node-centered data. Interfaces for elliptic equation solvers are described in chapter 4 . Some internal classes of the solvers are described in chapter 5. User interfaces for physical boundary conditions are described in chapter 6 .

The AMRNodeElliptic package requires that Chombo library be installed. See chapter 1 of the Chombo design document [CGL ${ }^{+} 00$ ] for requirements and installation of Chombo.

\subsection{Constructing a makefile with AMRNodeElliptic}

This section specifies how to construct a GNUmakefile compatible with the AMRNodeElliptic extension to Chombo. Most of this is also described in section 1.2.3 in the Chombo design document $\left[\mathrm{CGL}^{+} 00\right]$.

To make an external makefile, first say where Chombo is installed by specifying CHOMBO_HOME and the base name of the application by specifying ebase.

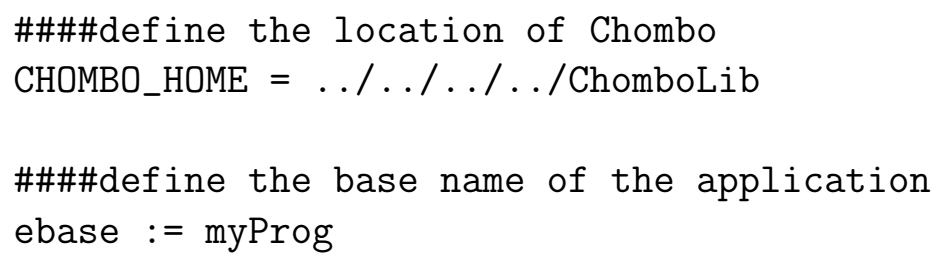

Then specify the names of the Chombo libraries that should be linked:

\#\#

\#\# names of Chombo libraries needed by this program, in order of search. 
\#\#

LibNames $:=$ AMRTools BoxTools

Now specify the path and source files external to Chombo and AMRNodeElliptic:

\#\# path to look for source files not in Chombo

VPATH_LOCAL $=$.

INCLUDES_LOCAL $=-\mathrm{I}$.

\#\# C++ sources go here

CXX_SOURCES $=$ NodeMaskPoissonBC.cpp testFuncs. cpp testRunPoint.cpp

\#\# Chombo Fortran sources go here

FORT_SOURCES $=$ testProb.ChF OutsideSphereMask. ChF

\#\# standard $\mathrm{C}$ sources go here

C_SOURCES $=$ myc1.c myc2.c

\#\# standard Fortran sources go here

F_SOURCES = myfort1.f myfort2.f

So far, the description of the makefile is as for any makefile that uses the Chombo library. When using AMRNodeElliptic, end the makefile with the following lines:

\#\#\#\#define location of AMRNodeElliptic source directory

SRC_DIR $=\ldots / \ldots /$ src

\#\# these commands include the AMRNodeElliptic source

include \$(SRC_DIR)/Make.package

INCLUDES_LOCAL $+=-$ I\$ (SRC_DIR)

VPATH_LOCAL $+=\$($ SRC_DIR)

\#\# end of makefile for use with Chombo

include \$(CHOMBO_HOME)/mk/Make. example 


\section{Chapter 2}

\section{Subroutines Needed for Non-Rectangular Domains}

For non-rectangular domains, the user must supply two Fortran subroutines described in the AMRNodeElliptic design document [McC02]:

- reachablenodes specifies the geometry;

- nodalcoefficients specifies the coefficients of the operator.

\subsection{The reachablenodes subroutine}

This subroutine should return an array telling which nodes are in the domain and which are reachable from their neighbors along lines parallel to the coordinate axes, where "reachable" in this context means that the connecting segment lies entirely in the domain. We represent the domain by $\Omega$.

subroutine reachablenodes(CHF_CONST_REAL $[\mathrm{dx}]$, CHF_FIA[mask])

Arguments:

- $\mathrm{dx}$ is the mesh spacing.

- mask is node-centered and has $2 \mathbf{D}+1$ components.

At any node index $i$ of the underlying Box of mask, the corresponding point in space is $\boldsymbol{x}=\boldsymbol{x}_{0}+\left(\boldsymbol{i}-\frac{1}{2} \boldsymbol{u}\right) \cdot \mathrm{dx}$, and the components of mask should be set to 0 or 1 as follows (see Figure 2.1).

- component 0 is set to 1 if $\boldsymbol{x} \in \Omega$, and 0 otherwise.

- for each $d=0, \ldots, \mathbf{D}-1$, component $2 d+1$ is set to 1 if the entire segment $\left[\boldsymbol{x}, \boldsymbol{x}+\boldsymbol{e}^{d} \cdot \mathrm{dx}\right] \subset \Omega$, and 0 otherwise. 
- for each $d=0, \ldots, \mathbf{D}-1$, component $2 d+2$ is set to 1 if the entire segment $\left[\boldsymbol{x}, \boldsymbol{x}-\boldsymbol{e}^{d} \cdot \mathrm{dx}\right] \subset \Omega$, and 0 otherwise.
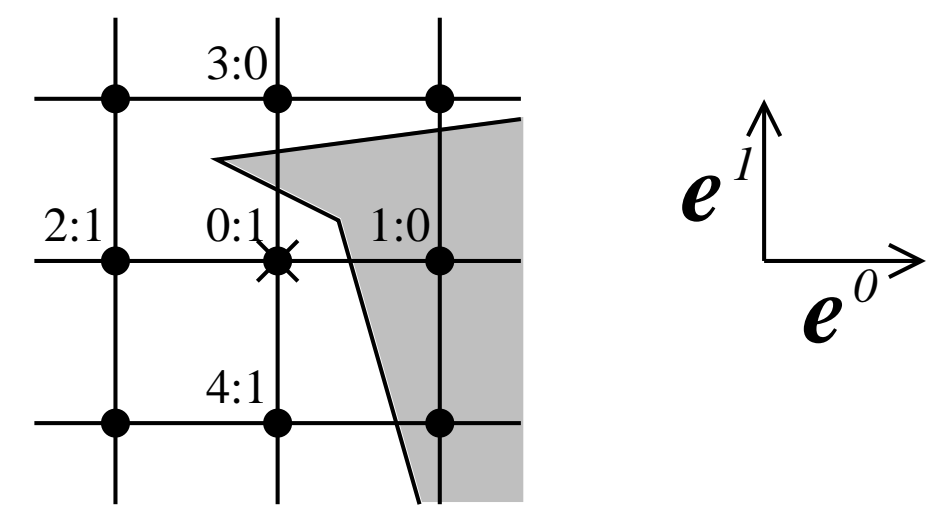

Figure 2.1: Components of the mask array at the center node indicated by $\times$, in two dimensions. The notation $i: v$ beside a node indicates that at node $\times$, component $i$ has value $v$, as determined by the reachability of the labeled node from $\times$. The shaded area is outside the domain. We have $v=1$ if the node is reachable from $\times$, and $v=0$ if it is not. Note that the point at top center is considered to be not reachable from $\times$, even though it is in the domain, because the connecting segment does not lie entirely in the domain.

\subsection{The nodalcoefficients subroutine}

This subroutine should return an array containing the coefficients of the discrete operator at each point.

subroutine nodalcoefficients(CHF_CONST_REAL[dx], CHF_FRA[coeffs])

Arguments:

- $\mathrm{dx}$ is the mesh spacing.

- coeffs is node-centered and has $2 \mathbf{D}+2$ components.

At any node index $i$ of the underlying Box of coeffs, the components of coeffs are set to the coefficients of the stencil for node $i$, as follows:

- component 0 is the coefficient of the value with index $\boldsymbol{i}$.

- for each $d=0, \ldots \mathbf{D}-1$, component $2 d+1$ is the coefficient of the value with index $\boldsymbol{i}+\boldsymbol{e}^{d}$. 
- for each $d=0, \ldots \mathbf{D}-1$, component $2 d+2$ is the coefficient of the value with index $\boldsymbol{i}-\boldsymbol{e}^{d}$.

- component $2 \mathrm{D}+1$ is the constant coefficient.

Summarizing, if $c_{\boldsymbol{i}}^{0}, \ldots c_{\boldsymbol{i}}^{2 \mathbf{D}+1}$ are the coefficients for node $\boldsymbol{i}$ at a level with mesh spacing $h$, then the operator at point $i$ is

$$
c_{\boldsymbol{i}}^{0} \cdot \varphi_{\boldsymbol{i}}^{N, l}+\sum_{d=0, \ldots, \mathbf{D}-1}\left(c_{\boldsymbol{i}}^{2 d+1} \cdot \varphi_{\boldsymbol{i}+\boldsymbol{e}^{d}}^{N, l}+c_{\boldsymbol{i}}^{2 d+2} \cdot \varphi_{\boldsymbol{i}-\boldsymbol{e}^{d}}^{N, l}\right)+c_{\boldsymbol{i}}^{2 \mathbf{D}+1} .
$$




\section{Chapter 3}

\section{Node-Centered Data}

\subsection{The class NodeFArrayBox}

The NodeFArrayBox class is a wrapper for a node-centered FArrayBox. This class is used in LevelData<NodeFArrayBox> with an underlying cell-centered DisjointBoxLayout.

The reason for introducing a new NodeFArrayBox class, instead of just using nodecentered FArrayBoxes, is to allow the use of LevelData. A LevelData<FArrayBox> based on node-centered FArrayBoxes would have an underlying DisjointBoxLayout of node-centered boxes, and no two of the boxes could be adjacent, because if they were, they would share points, and the layout would not be disjoint. To get around this problem, we use a cell-centered DisjointBoxLayout and build a LevelData<NodeFArrayBox> on it, with the data located at the surrounding nodes of the cell-centered grids. Data at nodes shared by two or more grids is stored redundantly in all of the corresponding NodeFArrayBoxes.

The API for NodeFArrayBox is as follows.

- NodeFArrayBox ()

Default constructor. User must subsequently call define.

- Constructor.

$\begin{array}{ll}\text { NodeFArrayBox } & \\ \text { const Box\& } & \text { a_bx, } \\ \text { int } & \text { a_nComp }=1)\end{array}$

Creates a NodeFArrayBox object.

Inputs:

- a_bx specifies the cell-centered box. The data lie on the surrounding nodes of this box.

- a_nComp specifies the number of components of the FArrayBox. 
- define(

$$
\begin{array}{ll}
\text { const Box\& } & \text { a_bx } \\
\text { int } & \text { a_nComp }=1)
\end{array}
$$

Defines a NodeFArrayBox object. Arguments are the same as those of the constructor above.

- FArrayBox\& getFab()

Returns a reference to the node-centered FArrayBox containing the data.

- const Box\& box()

Returns the cell-centered box of which the surrounding nodes contain the data.

- Real norm(

$$
\begin{aligned}
& \text { const Real a_dx, } \\
& \text { const int a_p }=2, \\
& \text { const int a_startComp }=0 \text {, } \\
& \text { const int a_numComp }=1 \text { ) const }
\end{aligned}
$$

For a_p $>0$, returns the $L^{\text {a-p }}$ norm of the data in a_numComp components, starting at component number a_startComp. The norm is computed by integration using the trapezoidal rule with mesh spacing $a_{-} d x$, over those nodes that are in the domain as determined by calling reachablenodes. For a_p $=0$, returns the $L^{\infty}$ norm of the same data. An overloaded version of this function performs its operations over a subbox. Another overloaded version takes as an argument the mask array returned from reachablenodes, so that reachablenodes need not be called in this function.

- Real maxnorm(

const int a_startComp $=0$, const int a_numComp $=1$ ) const

Returns the maximum value of the data in a numComp components, starting at component number a_startComp, in those nodes that are in the domain as determined by calling reachablenodes. An overloaded version of this function performs its operations over a subbox. Another overloaded version takes as an argument the mask array returned from reachablenodes, so that reachablenodes need not be called in this function.

\section{Principal data members}

- Box m_box. The cell-centered box of which the surrounding nodes contain the data.

- FArrayBox m_fab. The node-centered data. 


\subsection{Notes on LevelData<NodeFArrayBox $>$ : : copyTo}

The API for LevelData<NodeFArrayBox> is described in the Chombo design document $\left[\mathrm{CGL}^{+} 00\right]$, but one part of it deserves particular attention because it does not work in the same way.

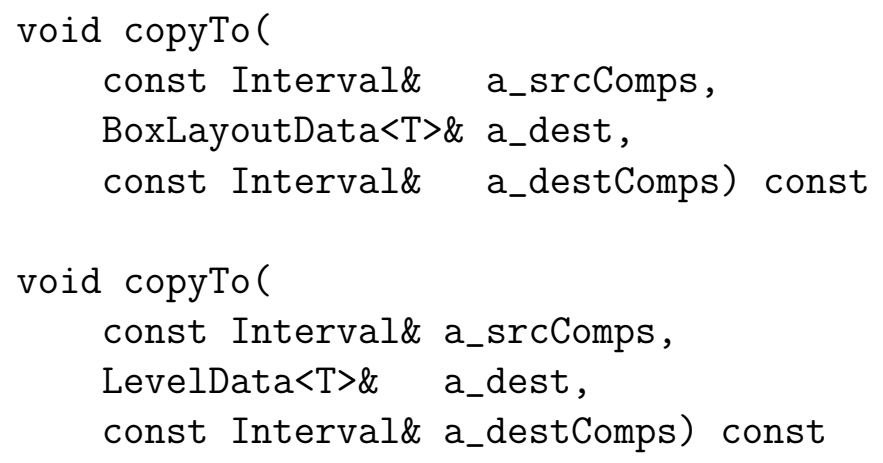

The LevelData<NodeFArrayBox>: : copyTo function must be used with caution. If the source LevelData and the destination, a_dest, do not have the same underlying grid layout, then it is possible that some data will not be copied from the source to the destination. The reason is that, in determining points where data should be copied, copyTo finds intersections of the underlying cell-centered boxes and then copies at the surrounding nodes. See Figure 3.1 for an example in which data are not copied at some of the nodes shared by source and destination.

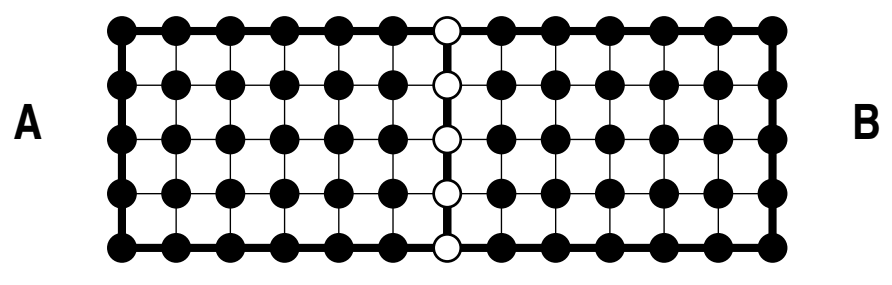

Figure 3.1: Suppose A and B are LevelData<NodeFArrayBox $>$ es, each having an underlying grid layout of a single grid, and these two grids abut, as shown above where the nodes of intersection are indicated by hollow circles. Then the statement A. copyTo(A.interval (), B, B.interval ()) results in no data being copied from A to $B$, even though they share nodes, because the two grids have no cells in common.

If all cells in the grid layout of the destination of the copyTo are also in the grid layout of the source, then this problem of uncopied data does not arise. For example, when copying all of the data on one AMR level to a coarsened version of the grids on a finer AMR level, copyTo works as expected, because of the nesting requirement for AMR levels.

But whenever we want to use LevelData<NodeFArrayBox>: : copyTo from src where the underlying grid layout of the destination dest may contain cells that are not in the grid layout of this, we use a Copier object, as follows: 
Copier myCopier(src.getBoxes(), dest.getBoxes(), IntVect::Unit); src.copyTo(srcComps, dest, destComps, myCopier);

\subsection{Node-centered norms}

\subsubsection{Norms of valid data on a single level}

- Real norm(const LevelData<NodeFArrayBox>\& a_phi,

const ProblemDomain\&

const DisjointBoxLayout*

const int

const Real

const Interval

const int

bool a_domain,

a_finerGridsPtr, a_nRefFine,

a_dx,

a_comps,

a_p,

a_verbose $=$ true)

- Real norm(const LevelData<NodeFArrayBox>\& a_phi,

const Box\&

const DisjointBoxLayout*

const int

const Real

const Interval

const int

bool a_domain,

a_finerGridsPtr, a_nRefFine,

a_dx, a_comps,

a_p,

a_verbose $=$ true)

Returns the norm of valid data on one level.

Inputs:

- a_phi is the data at this level.

- a_domain is the entire cell-centered problem domain at this level.

- a_finerGridsPtr is a pointer to the grid layout at the next finer level, or NULL if there is no finer level.

- a_nRefFine is the refinement ratio between this level and the next finer level.

- a_dx is the mesh spacing at this level.

- a_comps specifies the components over which to take the norm.

- a_p specifies which norm to take: if a_p $=0$ then the $\max \left(L^{\infty}\right)$ norm, and if a_p $>0$ then the $L^{\mathrm{a}-\mathrm{p}}$ norm.

- a_verbose specifies whether to display the norm of each grid.

This function calls reachablenodes. An overloaded version takes as an argument the mask array returned from reachablenodes, so that reachablenodes need not be called in this function. 
Other overloaded versions of this function include arguments with the exterior boundary nodes of this level, and the interior boundary nodes of the coarsened finer-level grids.

- Real maxnorm(const LevelData<NodeFArrayBox $>\&$ a_phi,

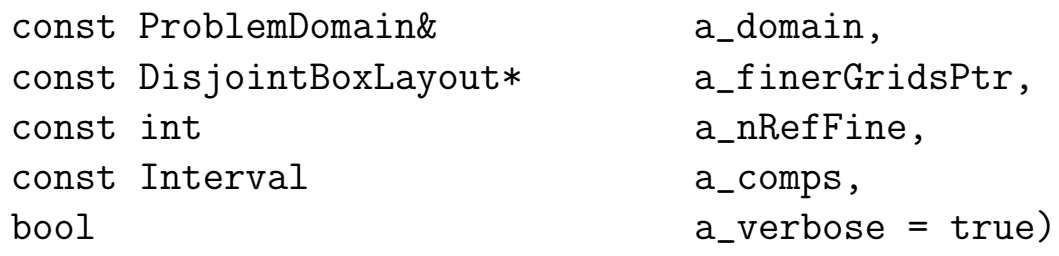

- Real maxnorm(const LevelData<NodeFArrayBox>\& a_phi,

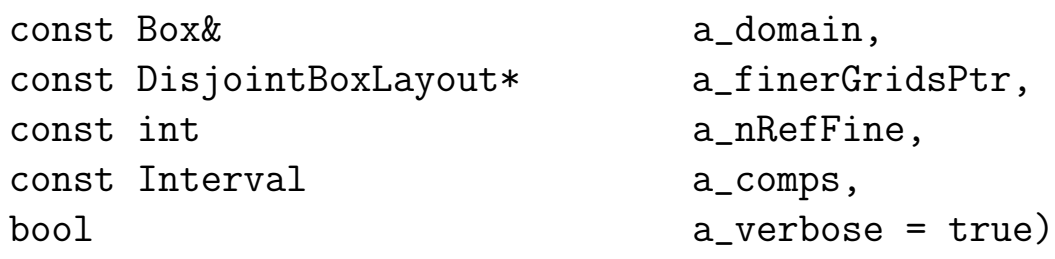

Returns the $\max \left(L^{\infty}\right)$ norm of valid data on one level. Inputs are a subset of those for the norm function above, and there are overloaded versions as with norm.

\subsubsection{Norms of data on multiple levels}

- Real norm(

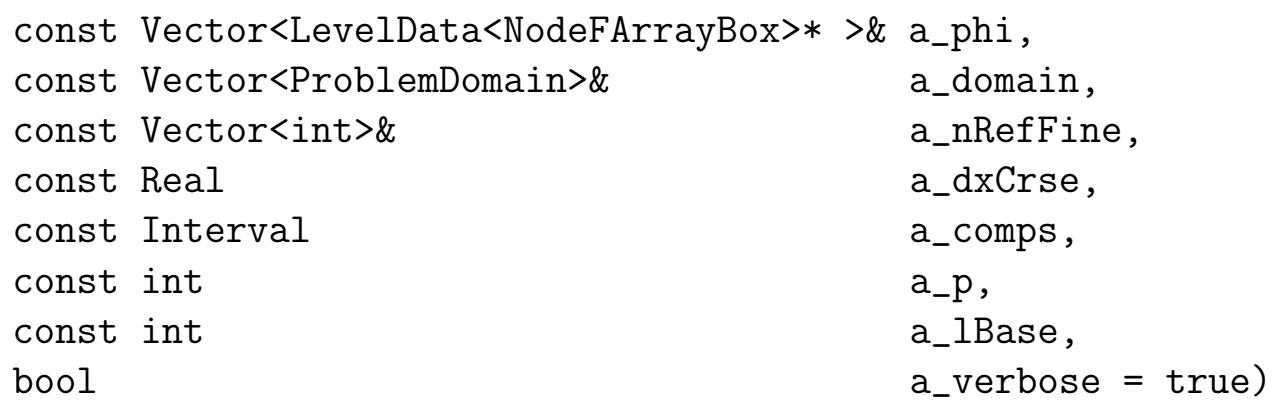

- Real norm(

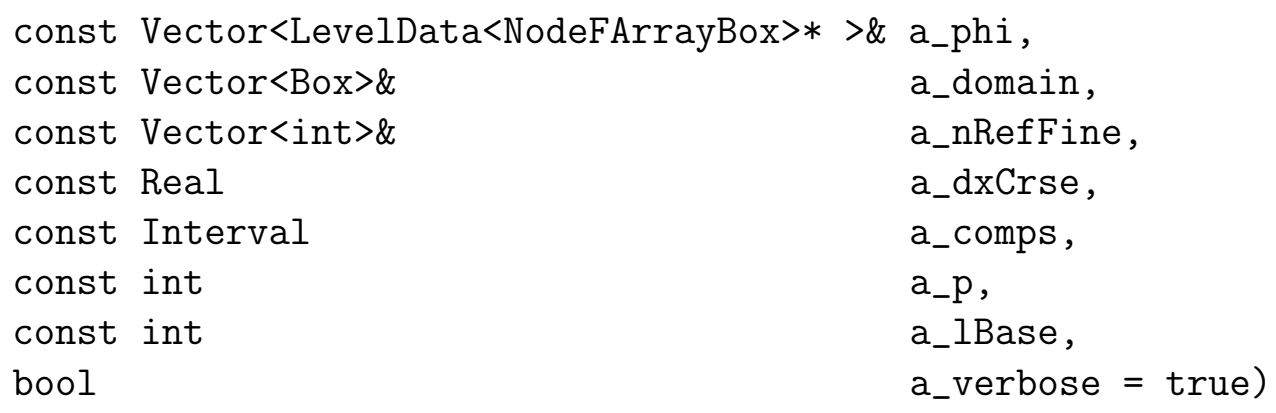

Returns the norm of valid data on multiple levels.

Inputs: 
- a_phi is a vector of pointers to data at multiple levels.

- a_domain specifies the entire cell-centered problem domain at all levels of resolution. Vector index corresponds to level number.

- annRefFine specifies the refinement ratios between adjacent levels. a_nRefFine [ilev] is the refinement ratio between levels ilev and $i l e v+1$. a_nRefFine [ilev] must be a power of two, with a_nRefFine[ilev] $\geq 1$.

- a_dxCrse is the mesh spacing at level a_lBase.

- a_comps specifies the components over which to take the norm.

- a_lBase is the index of the coarsest level on which the norm is to be computed.

- a_p specifies which norm to take: if a_p $=0$ then the $\max \left(L^{\infty}\right)$ norm, and if a_p $>0$ then the $L^{\text {a-p }}$ norm.

- a_verbose specifies whether to display the norm of each grid.

- Real maxnorm(

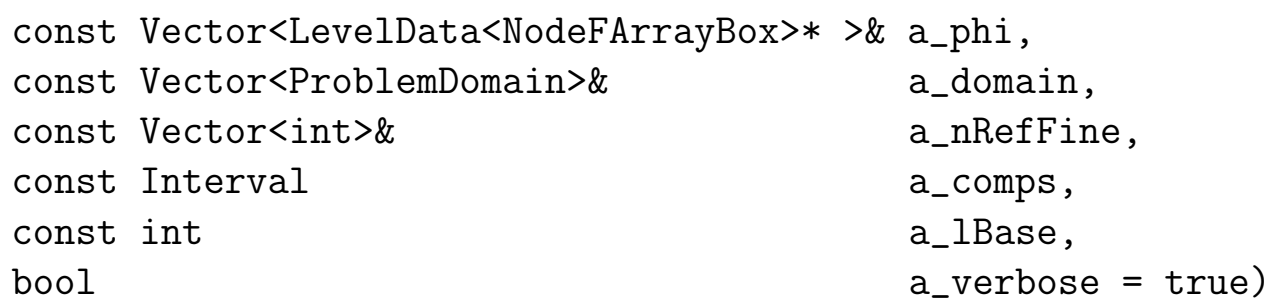

- Real maxnorm(

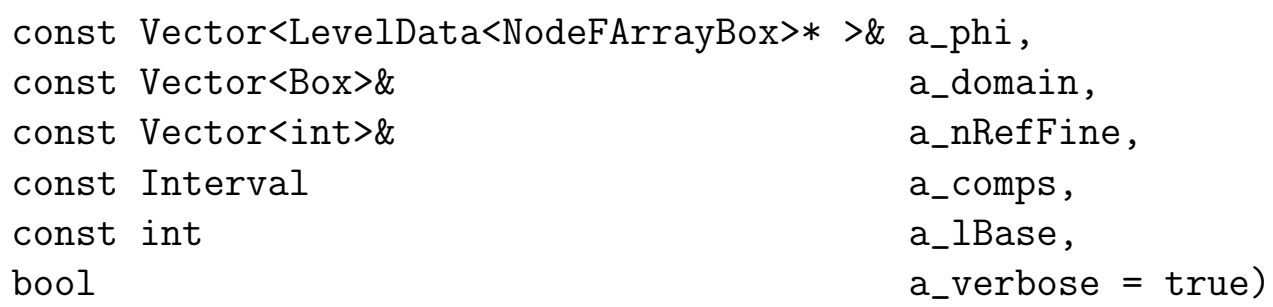

Returns the $\max \left(L^{\infty}\right)$ norm of valid data on multiple levels. Inputs are a subset of those for the multi-level norm function above.

\subsection{Dot product for node-centered data}

The DotProductNodes functions return the dot product of data distributed over two instances of LevelData $<$ NodeFArrayBox $>$ with the same layout. The dot product is the sum, over all valid nodes and over specified components, of the product of the data in the two data holders. That is, for data sets $f$ and $g$ on nodes $\Omega_{N}$, the dot product is

$$
f \cdot g=\sum_{i \in \Omega_{N, v a l i d}} f_{i} g_{i} .
$$


- Real DotProductNodes (

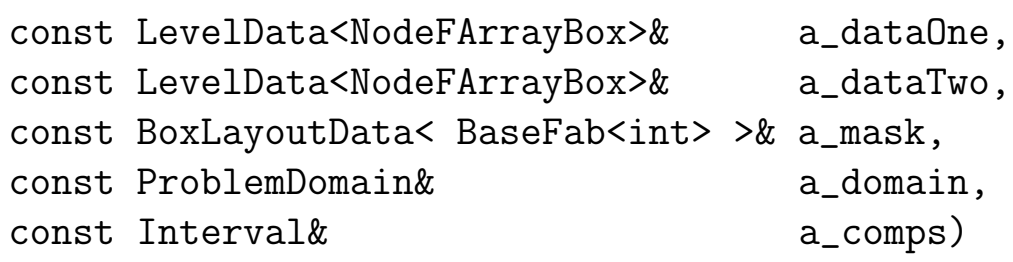

- Real DotProductNodes (

const LevelData $<$ NodeFArrayBox $>\& \quad$ a_dataOne,

const LevelData $<$ NodeFArrayBox $>\&$ a_dataTwo,

const BoxLayoutData< BaseFab<int $>>\&$ a_mask,

const Box\& a_domain,

const Interval\& a_comps)

Returns the dot product of data on one level.

Inputs:

- a_dataOne contains the first set of data on a level.

- a_dataTwo contains the second set of data on a level.

- a mask is the mask array from REACHABLENODES on this level.

- a_domain is the cell-centered problem domain on this level.

- a_comps specifies the components to be used in computing the dot product.

An overloaded version of this function includes the exterior boundary nodes of the level as an argument. It is more efficient to use this other version when the dot product is to be computed multiple times on data with the same layout.

\subsection{Functions for interior and exterior boundary nodes}

An interior node of a grid layout is any node in the layout with the property that all of the cells adjacent to the node are also contained in the grid layout. An interior boundary node of a grid layout is an interior node that lies on the boundary of one or more grids in the layout. See Figure 3.2 for a sample layout with interior boundary nodes marked.

An exterior node or exterior boundary node of a grid layout is any node of the layout that is not an interior node. See Figure 3.3 for the same layout as Figure 3.2, with exterior nodes marked.

\subsection{1 interiorBoundaryNodes functions}

The interiorBoundaryNodes functions return the interior boundary nodes of a DisjointBoxLayout. The interface would be simplest if the function returned a reference to an IntVectSet, but because of the way that IntVectSet is implemented, 


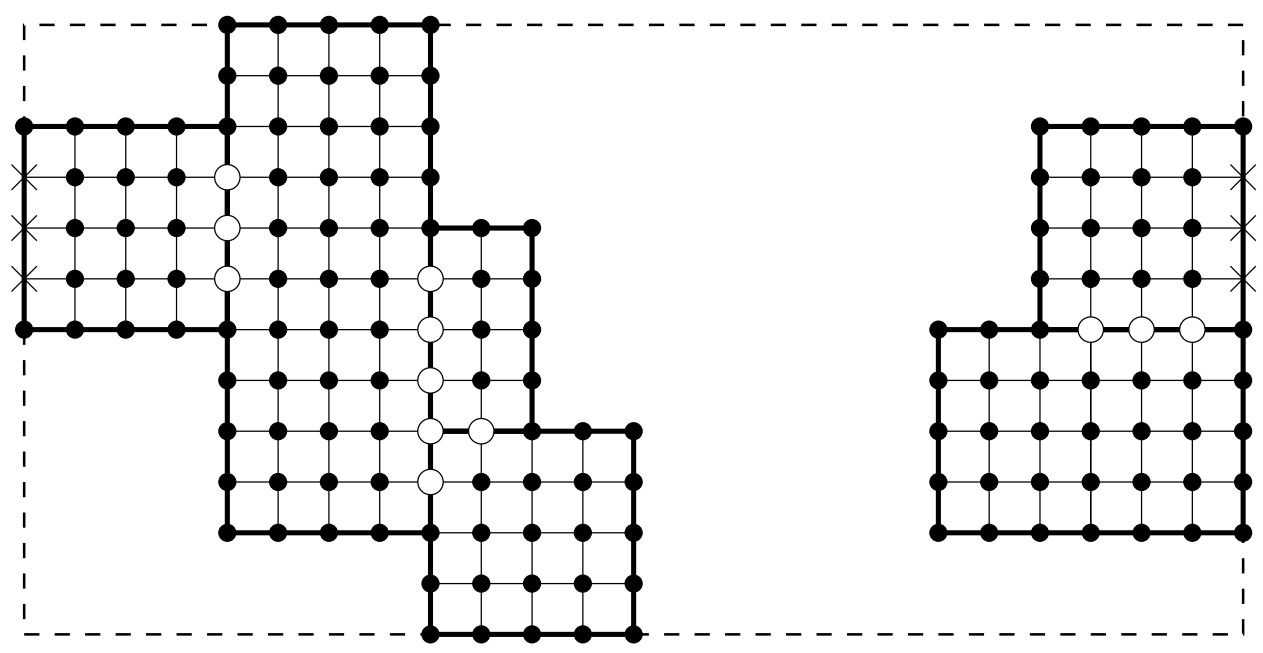

Figure 3.2: Hollow circles indicate the interior boundary nodes of a two-dimensional grid layout inside a problem domain delineated by the dashed lines. Nodes marked $\times$ are also interior boundary nodes if the problem domain is periodic in the horizontal direction.

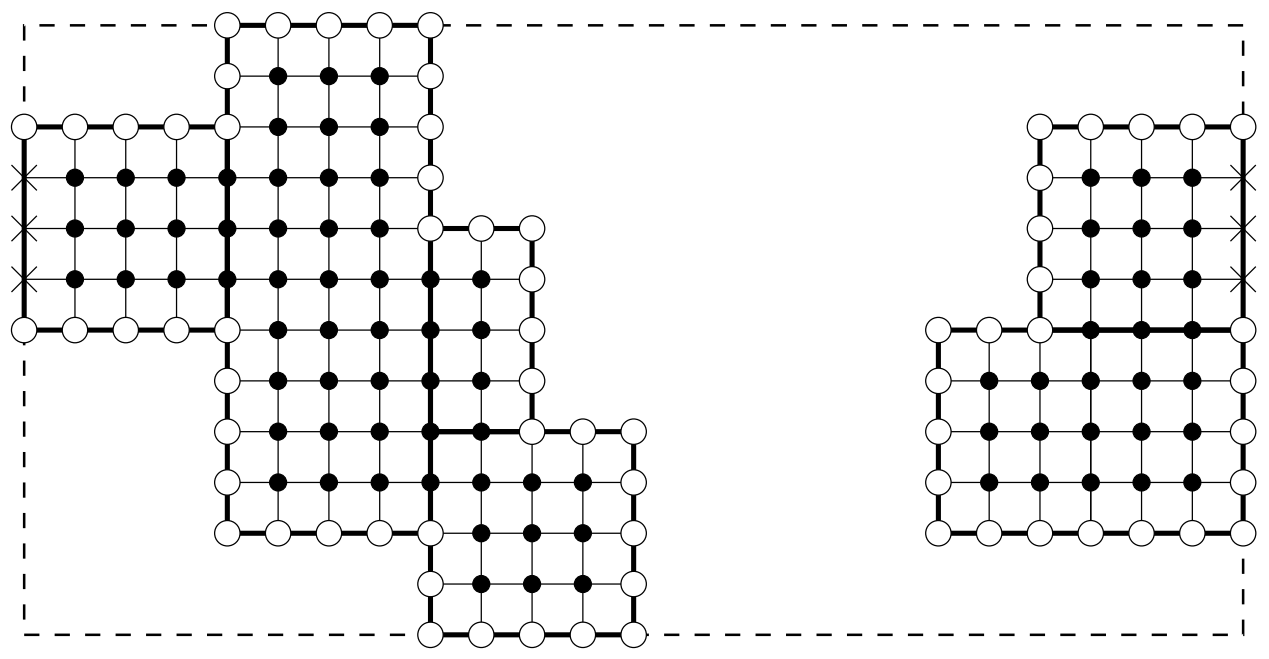

Figure 3.3: Hollow circles indicate the exterior boundary nodes of a two-dimensional grid layout inside a problem domain delineated by the dashed lines. Nodes marked $\times$ are also exterior boundary nodes if the problem domain is not periodic in the horizontal direction. Any node that is not an exterior boundary node is an interior node. 
this would be very slow. Instead, the first interiorBoundaryNodes function returns a reference to a LayoutData< Vector<IntVectSet> > such that the union of all the IntVectSets in the Vectors contains exactly the indices of all interior boundary nodes of the input layout.

To make matters even more complicated, there is a second interiorBoundaryNodes function, which takes two DisjointBoxLayout arguments. This function returns a reference to a LayoutData that stores the indices of interior boundary nodes of one layout that are also nodes (interior or exterior) of a second layout. The output of this function is needed for calling copyInteriorNodes, described in section 3.5.3.

- void interiorBoundaryNodes (

LayoutData< Vector<IntVectSet> $>\&$ a_IVSV, const DisjointBoxLayout\& a_boxes,

const ProblemDomain\& a_domain)

- void interiorBoundaryNodes (

LayoutData< Vector<IntVectSet> > \& a_IVSV, const DisjointBoxLayout\& a_boxes,

const Box\& a_domain)

Returns the interior boundary nodes of a layout.

Inputs:

- a_boxes specifies the cell-centered layout of boxes.

- a_domain specifies the cell-centered problem domain that contains the layout.

\section{Output:}

- a_IVSV contains the interior boundary nodes of a_boxes in a LayoutData based on a_boxes. For each box in the layout of a_boxes, there is a Vector of IntVectSets that together contain the indices of the interior boundary nodes of the box.

- void interiorBoundaryNodes(

LayoutData< Vector<IntVectSet> >\& a_IVSV, const DisjointBoxLayout\& a_dest,

const DisjointBoxLayout\& a_src,

const ProblemDomain\& a_domain)

- void interiorBoundaryNodes (

LayoutData< Vector<IntVectSet> > \& a_IVSV,

const DisjointBoxLayout\& a_dest,

const DisjointBoxLayout\& a_src,

const Box\& a_domain) 
Returns the interior boundary nodes of a source layout that are also nodes of a destination layout. This function is used when copying from data based on the source layout to data based on the destination layout.

Inputs:

- a_dest specifies the cell-centered layout of boxes on which to base the LayoutData to be returned.

- a_src specifies the cell-centered layout of boxes of which we wish to find the interior boundary nodes.

- a_domain specifies the cell-centered problem domain that contains both layouts.

\section{Output:}

- a_IVSV contains the interior boundary nodes of a_src in a LayoutData based on the layout in a_dest. For each box in the layout of a_dest, there is a Vector of IntVectSets that together contain the indices of all nodes of the box that are also interior boundary nodes of a_src.

If a_dest is the same as a_src, then the result is the same as calling the first interiorBoundaryNodes function with a_boxes set to a_dest.

\subsection{2 exteriorBoundaryNodes function}

- void exteriorBoundaryNodes (

$$
\begin{array}{ll}
\text { LayoutData< Vector }<\text { IntVectSet }>>\& & \text { a_exterior, } \\
\text { const LayoutData< Vector<IntVectSet }>>\& & \text { a_interior, } \\
\text { const DisjointBoxLayout\& } & \text { a_boxes })
\end{array}
$$

Returns the exterior boundary nodes (also called exterior nodes) of a_boxes.

\section{Inputs:}

- a_interior is the object returned by interiorBoundaryNodes (a_interior, a_boxes, a_domain) described in section 3.5.1.

- a_boxes specifies the cell-centered layout of boxes.

\section{Output:}

- a_exterior contains the exterior boundary nodes of a_boxes in a LayoutData based on a_boxes. For each box in the layout of a_boxes, there is a Vector of IntVectSets that together contain the indices of the exterior boundary nodes of the box. 


\subsection{3 copyInteriorNodes function}

The copyInteriorNodes function copies data from a source to a destination, but only from interior nodes of the source. Recall that the interior nodes are simply those that are not exterior nodes. This function is similar to copyTo but copies valid data only. See Figure 3.4 for an example.

- void copyInteriorNodes (

$$
\begin{array}{ll}
\text { LevelData }<\text { NodeFArrayBox }>\& & \text { a_dest, } \\
\text { const LevelData }<\text { NodeFArrayBox }>\& & \text { a_src, } \\
\text { const LayoutData }<\text { Vector }<\text { IntVectSet }>>\& & \text { a_IVSV) }
\end{array}
$$

Copies data from the interior boundary nodes of a_src to a_dest.

Inputs:

- a_src contains the data to be copied at its interior nodes.

- a_IVSV is the object returned by

$$
\begin{array}{r}
\text { interiorBoundaryNodes(a_interior, a_dest.boxLayout(), } \\
\text { a_src.boxLayout(), a_domain) }
\end{array}
$$

described in section 3.5.1.

\section{Output:}

- a_dest, at interior nodes of the layout of a_src, is replaced by data from a_src. Elsewhere, a_dest is unchanged.

\subsection{4 zeroBoundaryNodes function}

- void zeroBoundaryNodes (

$$
\begin{array}{lr}
\text { BoxLayoutData }<\text { NodeFArrayBox }>\& & \text { a_dest, } \\
\text { const LayoutData }<\text { Vector }<\text { IntVectSet }>>\& \text { a_IVSV) }
\end{array}
$$

Sets data to zero on specified nodes.

\section{Input:}

- a_IVSV is an object containing indices of nodes for each box, where data are to be set to zero. Usually it stores the indices of exterior boundary nodes of a_dest. These are obtained with the sequence of calls:

interiorBoundaryNodes (a_IVSVint, a_dest.boxLayout (), a_domain) ; exteriorBoundaryNodes(a_IVSV, a_IVSVint, a_dest.boxLayout());

where a_domain is the cell-centered problem domain.

\section{Output:}



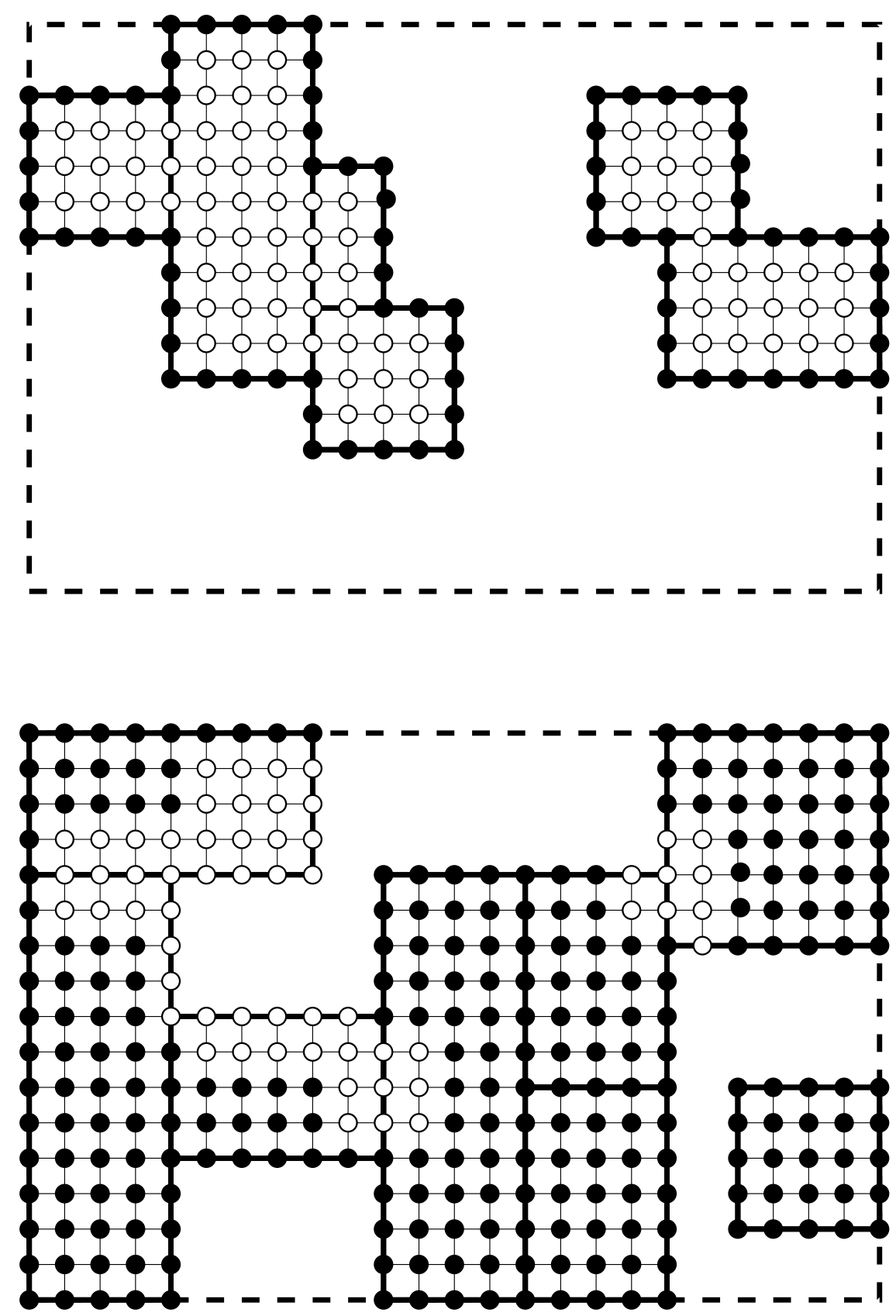

Figure 3.4: An example of a source and destination layout for copyInteriorNodes. The problem domain, delineated by dashed lines, is taken to be non-periodic.

Top: Hollow circles indicate the interior nodes of a source layout.

Bottom: Hollow circles indicate the nodes of a destination layout that are filled by copyInteriorNodes with data from the source layout above. 
- a_dest is set to zero at nodes in a_IVSV. Elsewhere, a_dest is unchanged.

The underlying layouts of a_dest and a_IVSV must be the same. 


\section{Chapter 4}

\section{Interface for AMRNodeElliptic Solver}

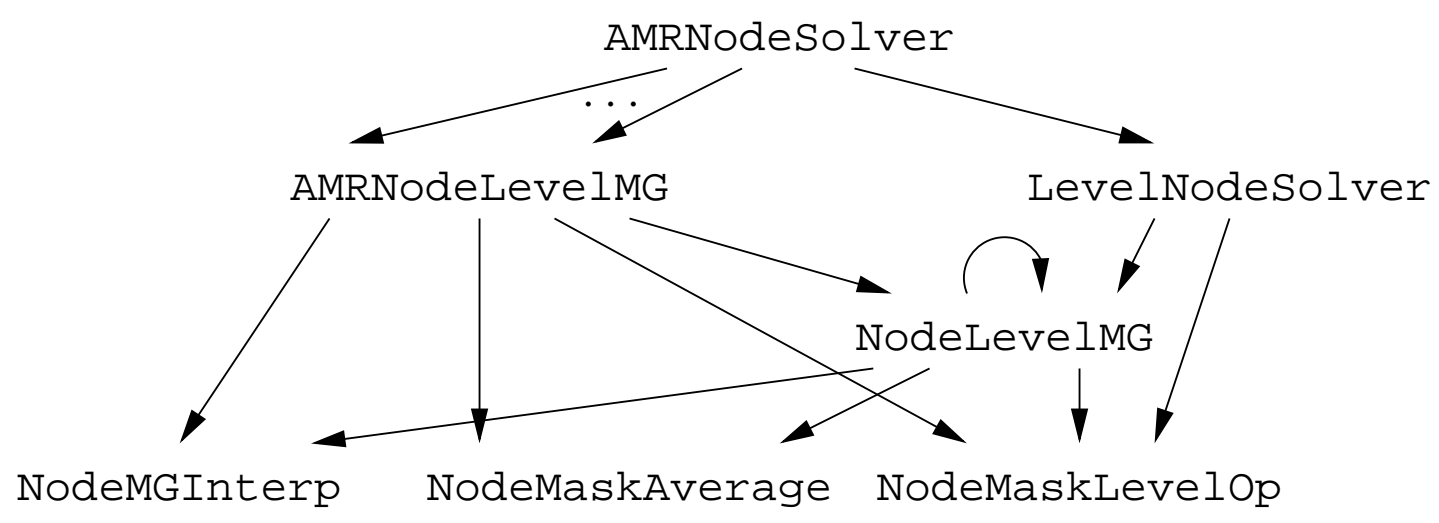

Figure 4.1: This chart shows the major classes of AMRNodeElliptic used by AMRNodeSolver. An arrow from one class to another indicates that an instance of the first class defines an instance of the second class. In each case, only one instance of the second class is defined, except that the AMRNodeSolver defines an instance of AMRNodeLevelMG for each level in the AMR hierarchy.

\subsection{The class AMRNodeSolver}

The AMRNodeSolver class solves an elliptic equation on an adaptive mesh refinement hierarchy of node-centered grids. The boundary conditions are obtained from a combination of interpolation from the next coarser level of grids, plus some set of physical boundary conditions on a rectangular domain.

The API is as follows.

- void define(

const Vector<DisjointBoxLayout>\& a_gridsLevel,

const Vector<ProblemDomain $>\&$ a_domainLevel, 


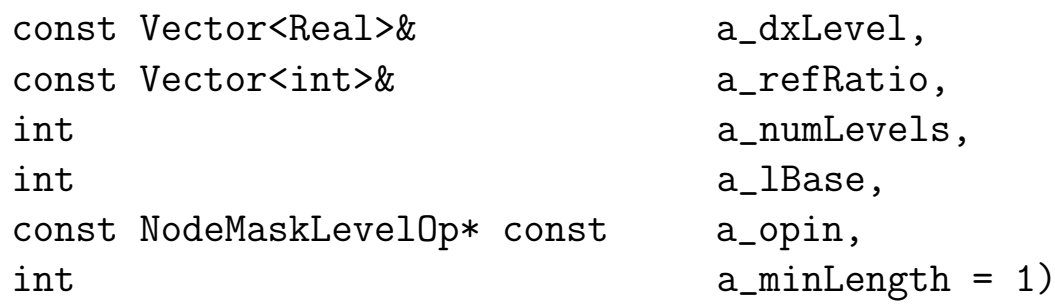

- void define(

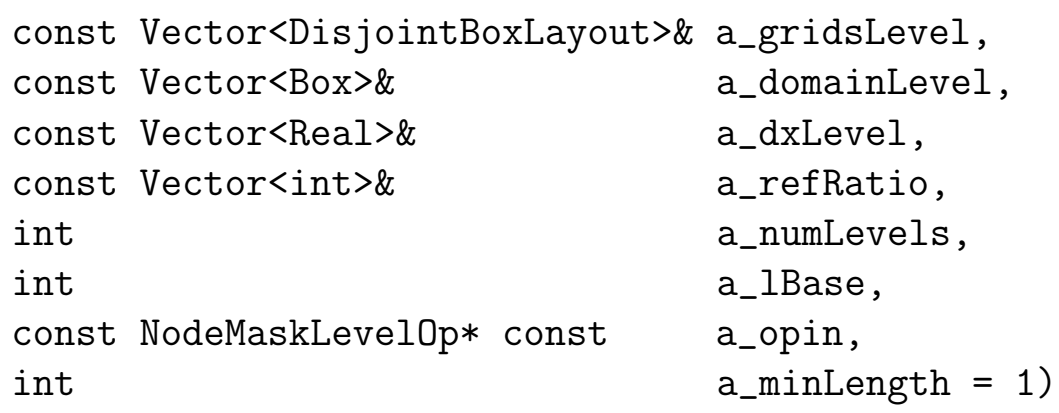

Sets up the internal state of an AMRNodeSolver object with information about the grid hierarchy and the operator.

\section{Inputs:}

- a_gridsLevel specifies the layout of the cell-centered grids on all levels. Vector index corresponds to level number.

- a_domainLevel specifies the entire cell-centered problem domain at all levels of resolution. Vector index corresponds to level number.

- a_dxLevel specifies the mesh spacing at all levels. Vector index corresponds to level number.

- a_refRatio specifies the refinement ratios between adjacent levels. a_refRatio [ilev] is the refinement ratio between levels ilev and ilev+1. a_refRatio[ilev] must be a power of two, with a_refRatio[ilev] $\geq 1$.

- a_numLevels is the number of AMR levels in the calculation. The length of the Vectors a_gridsLevel, a_domainLevel and a_dxLevel must be at least a_numLevels, and the length of the Vector a_refRatio must be at least a_numLevels-1.

- a_lBase is the index of the coarsest level on which the solution is to be computed.

- a_opin is a pointer to the NodeMaskLevelOp to use in solving.

- a_minLength is the minimum length of the maximally coarsened box in LevelNodeSolver, or 0 if there is to be no coarsening. If a minLength $\geq 1$ then the multigrid solver will coarsen boxes only so far as to maintain the lengths of all boxes as multiples of a_minLength. 
- void solveAMR(

$$
\begin{array}{ll}
\text { Vector }<\text { LevelData }<\text { NodeFArrayBox }>*>\& & \text { a_phiLevel, } \\
\text { const Vector }<\text { LevelData }<\text { NodeFArrayBox }>*>\& & \text { a_rhsLevel) }
\end{array}
$$

Solves the elliptic equation over the hierarchy of levels m_lBase ... $\mathrm{m}_{-}$finestLevel where $\mathrm{m}_{-}$finestLevel $=\mathrm{m} \_$numLevels -1 . If $\mathrm{m}_{-}$lBase $>0$, then the data at level $\mathrm{m}_{-}$lBase-1 is used to interpolate boundary conditions at boundary cells that are not adjacent to the domain boundary. Solves to tolerance m_tolerance.

\section{Inputs:}

- a_phiLevel contains pointers to the current guess at the solution values for levels $\left(\right.$ lMin $=\max \left(m_{-}\right.$lBase-1, 0) ) . m $\mathrm{m}_{-}$finestLevel. Index in the Vector corresponds to level number. These values are updated in place.

- a_rhsLevel contains pointers to values of the right-hand side for levels m_lBase .. m_finestLevel.

\section{Outputs:}

- a_phiLevel contains pointers to values of the updated solution at the same levels.

- void AMRVCycleMG(

Vector $<$ LevelData $<$ NodeFArrayBox $>*>\&$ a_phiLevel,

const Vector $<$ LevelData $<$ NodeFArrayBox $>*>$ \& a_rhsLevel)

Does one relaxation $\mathrm{V}$-cycle using an $\mathrm{AMR}$ multigrid solver.

Inputs:

- a_phiLevel contains pointers to current guess at values of the solution at levels m_lBase .. m_finestLevel. Vector index corresponds to level number.

- a_rhsLevel contains pointers to values of the right-hand side on the same levels as a_phiLevel.

\section{Outputs:}

- a_phiLevel contains pointers to values of the updated solution.

- Real computeResidualNorm(int a_normNtype)

Returns the norm of the multilevel residual on levels $m_{-}$lBase to $\mathrm{m}_{-}$finestLevel, where the residual at level ilev has been stored in m_amrmgLevel[ilev]->m_resid. The residual must have been computed already before this function is called.

Inputs:

- a_normType is the type of norm: 0 for max norm, or $\mathrm{p}>0$ for $L_{p}$ norm. 
- void applyAMROperator (

$$
\begin{array}{ll}
\text { LevelData<NodeFArrayBox }>\& & a_{-} \text {lofPhi, } \\
\text { Vector }<\text { LevelData<NodeFArrayBox }>*>\& & a_{-} \text {phiLev, } \\
\text { int } & \left.a_{-} \text {ilev }\right)
\end{array}
$$

Applies the multilevel AMR operator to the data on a level.

Inputs:

- a_phiLev contains pointers to current guess at values of the solution at levels m_lBase .. m_finestLevel. Vector index corresponds to level number.

- a_ilev is level on which operator is to be applied. The result depends on a_phiLev[a_ilev], as well as a_phiLev[a_ilev-1] and a_phiLev [a_ilev+1] if they are defined.

\section{Outputs:}

- a_lofPhi contains value of operator applied on valid region of level a_ilev.

- void computeAMRResidual (

$$
\begin{array}{ll}
\text { LevelData }<\text { NodeFArrayBox }>\& & \text { a_res, } \\
\text { Vector }<\text { LevelData }<\text { NodeFArrayBox }>*>\& & \text { a_phiLevel, } \\
\text { const Vector<LevelData<NodeFArrayBox }>*>\text { \& } & \text { a_rhsLevel, } \\
\text { int } & \text { a_ilev) }
\end{array}
$$

Computes the residual on a level using the multilevel operator.

\section{Inputs:}

- a_phiLevel contains pointers to current guess at values of the solution at levels $m_{-}$lBase .. m_finestLevel. Vector index corresponds to level number.

- a_rhsLevel contains pointers to the right-hand side at the same levels as a_phiLevel.

- a_ilev is level on which the residual is to be computed. The result depends on a_rhsLevel[a_ilev] and a_phiLevel[a_ilev], as well as a_phiLevel [a_ilev-1] and a_phiLevel[a_ilev+1] if they are defined.

\section{Outputs:}

- a_res contains residual on valid region of level a_ilev. This is also stored in m_amrmgLevel [ilev]->m_resid.

- void setTolerance(Real a_tolerance)

Sets the tolerance in the AMR solver: iterations end when the norm of the residual is less than a_tolerance times the initial residual. Default is $1.0 e-10$. 
- void setOperatorTolerance(Real a_operatorTolerance)

Sets the "operator tolerance" of the AMR solver: iterations end if the ratio of the new residual to the old residual exceeds $1-$ a_operatorTolerance and at least m.minIter iterations have been performed. Default is $1.0 e-5$.

- void setBottomTolerance(Real a_tolerance)

Sets the tolerance in the bottom solver, LevelNodeSolver m_levelSolver.

- void setMaxIter(int a_maxIter)

Sets the maximum number of iterations in the AMR solver. Default is 42 .

- void setMinIter(int a_minIter)

Sets the minimum number of iterations in the AMR solver before it will terminate due to lack of convergence. Default is 5 .

- void setBottomMaxIter(Real a_maxIter)

Sets the maximum number of iterations in the bottom solver, LevelNodeSolver::levelSolve or LevelNodeSolver::levelSolveH. Default is 33 .

- void setNumSmoothDown(int a_numSmoothDown)

Sets the number of iterations of smoothing for the downward part of the $\mathrm{V}$-cycle in NodeLevelMG: :mgRelax. Default is 4.

- void setNumSmoothUp(int a_numSmoothUp)

Sets the number of iterations of smoothing for the upward part of the $\mathrm{V}$-cycle in NodeLevelMG: :mgRelax. Default is 4.

- void setNumBottomGSRB (int a_numBottomGSRB)

Sets the number of iterations of smoothing at the bottom of the $V$-cycle in NodeLevelMG: :mgRelax. Default is 16.

- void setBottomSmoothing(bool a_doBottomSmooth)

Sets whether the smoother is applied at the bottom of the V-cycle in NodeLevelMG: : mgRelax. Default is true.

\section{Principal data members}

- Vector<AMRNodeLevelMG*> m_amrmgLevel is a vector of length m_numLevels containing pointers to AMRNodeLevelMG objects, each of which manages the data and operations on one level. 
- LevelNodeSolver m_levelSolver solves elliptic equations on level m_lBase using multigrid.

- Vector<DisjointBoxLayout> m_gridsLevel, Vector<Box>m_domainLevel, Vector<Real> m_dxLevel, Vector<int> m_refRatio, int m_numLevels, int $\mathrm{m}_{-}$lBase are set to the arguments a_gridsLevel, a_domainLevel, a_dxLevel, a_refRatio, a_numLevels, a_lBase in the constructor.

\subsubsection{Internal class AMRNodeLevelMG}

AMRNodeLevelMG manages the data and operations for AMRNodeSolver on one level. AMRNodeSolver defines a vector of AMRNodeLevelMG objects, one for each level. This class should be considered internal to AMRNodeSolver and should not be considered part of the Chombo API.

These functions are called from AMRNodeSolver. In a multigrid V-cycle, AMRNodeSolver: : AMRVCycleMG calls downSweep at each level from the finest down to one above the base level, solves at the base level with class LevelNodeSolver, and then calls upSweep at each level from one above the base up to the finest level.

- void define(

const AMRNodeSolver* const a_parent,

int a_level,

const NodeMaskLevelOp* const a_opin)

Sets up the internal state of an AMRNodeLevelMG object.

Inputs:

- a_parent is a pointer to the AMRNodeSolver object that called this constructor.

- a_level is the level for which this object manages data and operations.

- a_opin is a pointer to the NodeMaskLevelOp to use in solving.

- void applyAMROperator(

LevelData $<$ NodeFArrayBox $>\& \quad$ a_Lofphi,

Vector<LevelData<NodeFArrayBox $>*>\&$ a_phiLevel)

Calculates the multilevel AMR operator to the data on this level. This operator uses inhomogeneous coarse-fine boundary conditions and inhomogeneous physical domain boundary conditions.

Inputs:

- a_phiLevel contains pointers to current guess at values of the solution. Vector index corresponds to level number. The result depends on a_phiLev[m_level], as well as a_phiLev[m_level-1] and a_phiLev [m_level+1] if they are defined. 


\section{Outputs:}

- a_lofPhi contains value of operator applied on valid region of level m_level.

- void computeAMRResidual (

Vector $<$ LevelData $<$ NodeFArrayBox $>*>\& \quad$ a_phiLevel,

const Vector<LevelData<NodeFArrayBox $>*>$ \& a_rhsLevel)

Computes the residual on this level using the multilevel operator. The result is stored in local data m_resid.

\section{Inputs:}

- a_phiLevel contains pointers to current guess at values of the solution. Vector index corresponds to level number. The result depends on *a_phiLevel[m_level], as well as *a_phiLevel[m_level-1] and *a_phiLevel [m_level+1] if they are defined.

- a_rhsLevel contains pointers to the right-hand side at the same levels as a_phiLevel. The result depends on $*$ a_rhsLevel [m_level].

- void downSweep (

Vector $<$ LevelData<NodeFArrayBox $>*>\& \quad$ a_phiLevel,

const Vector $<$ LevelData<NodeFArrayBox $>*>\&$ a_rhsLevel)

Sweeps down a multigrid $\mathrm{V}$-cycle from this level to next coarser level. This function calculates the correction $\mathrm{m}_{-}$corr and updates the solution at this level with it. It also overwrites the residual at the next coarser level, if any, with the averaged residual at this level.

Inputs:

- a_phiLevel contains pointers to current guess at values of the solution. Vector index corresponds to level number.

- a_rhsLevel contains pointers to the right-hand side at the same levels as a_phiLevel.

\section{Outputs:}

- a_phiLevel contains pointers to updated solution.

- void upSweep(

Vector $<$ LevelData<NodeFArrayBox $>*>$ \& a_phiLevel, const Vector $<$ LevelData $<$ NodeFArrayBox $>*>$ \& a_rhsLevel)

Sweeps up a multigrid $\mathrm{V}$-cycle from the next coarser level to this level. This function interpolates the correction $\mathrm{m}_{-}$corr from the next coarser level, uses it to finish the calculation of residual m_resid, and updates the solution at this level.

Inputs: 
- a_phiLevel contains pointers to current guess at values of the solution. Vector index corresponds to level number.

- a_rhsLevel contains pointers to the right-hand side at the same levels as a_phiLevel.

\section{Outputs:}

- a_phiLevel contains pointers to updated solution.

- Real computeResidualNorm(int a_normNtype)

Returns the norm of internal data m_resid.

Inputs:

- a_normType is the type of norm: 0 for max norm, or $\mathrm{p}>0$ for $L_{p}$ norm.

\section{Principal data members}

- LevelData<NodeFArrayBox>m_resid is the residual calculated in computeAMRResidual.

- LevelData<NodeFArrayBox> $\mathrm{m}_{-}$corr is the correction at this level.

- int $\mathrm{m}_{-}$level is the level for which this object manages data and operations, set to a_level argument in constructor.

- NodeMaskLevelOp* m_levelOpPtr is the pointer to the level operator, set to a_opin argument in constructor.

- NodeMGInterp m.mginterp is used for interpolating the correction from the next coarser level to this level in upSweep.

- NodeMaskAverage $\mathrm{m}_{-}$average0p is used for averaging the residual down to the next coarser level in downSweep.

\subsubsection{Internal class NodeLevelMG}

The NodeLevelMG class is a multigrid solver on a level. It is used by the classes LevelNodeSolver and AMRNodeLevelMG (internal to AMRNodeSolver). This class should be considered internal to AMRNodeSolver and LevelNodeSolver, and should not be considered part of the Chombo API.

- void define(

const DisjointBoxLayout\& const DisjointBoxLayout* const ProblemDomain\& a_grids,

a_gridsCoarsePtr, a_domain, 


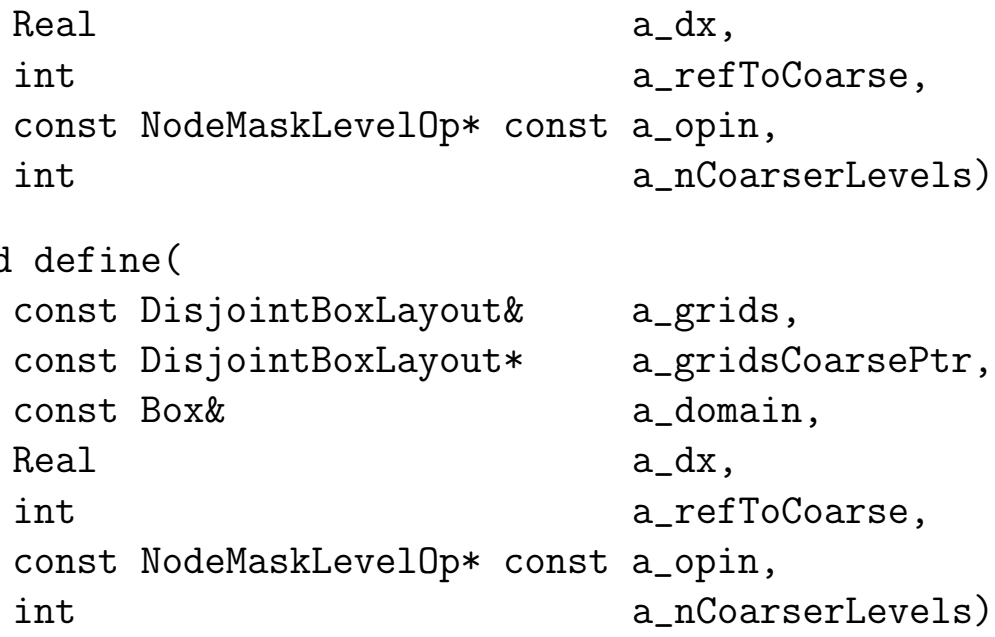

Defines the internal state of the NodeLevelMG object and allocates space for the residual. The arguments are the same as for the LevelNodeSolver constructor, but instead of a_minLength we have a_nCoarserLevels. This argument represents the number of coarser-level NodeLevelMG objects to be defined recursively. Namely,

- when defined from LevelNodeSolver,

* if a minLength is 0 then a nCoarserLevels is also 0;

$*$ if a_minLength $\geq 1$ then a_nCoarserLevels is the exponent of the largest power of 2 that divides the lengths of every grid at this level divided by a minLength. Thus after a nCoarserLevels coarsenings by 2 , the lengths of all grids will still be divisible by a minLength.

- when defined from AMRNodeLevelMG,

* if a minLength is 0 or a_level is 0 then a_nCoarserLevels is also 0;

* if a_minLength $\geq 1$ and a_level $\geq 1$ then a_nCoarserLevels $=$ $\log _{2}(r)-1$ where $r$ is the refinement ratio to the next coarser level.

- void mgRelax (

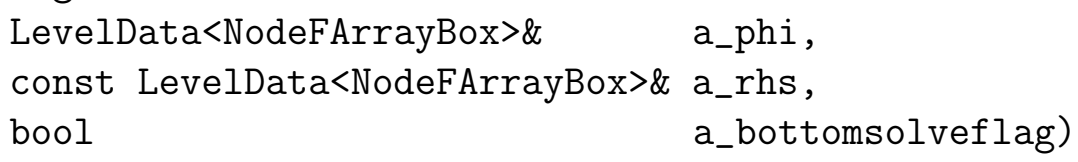

Invokes a relaxation step, updating a_phi with right-hand side a_rhs. It is assumed that the problem is in residual-correction form. In particular, only the homogeneous form of the physical and coarse-fine boundary conditions need be invoked. mgRelax calls itself recursively on the coarsened grids.

\section{Principal data members}

- LevelData<NodeFArrayBox> m_resid is the residual at this level. 
- LevelData<NodeFArrayBox> m_crseResid is the residual on the grids of this level coarsened by 2 .

- LevelData<NodeFArrayBox> m_crseCorr is the correction on the grids of this level coarsened by 2 .

- NodeMaskLevelOp* m_levelOpPtr is the pointer to the level operator, set to a_opin argument in constructor.

- NodeMaskAverage $\mathrm{m}$ average0p is used for averaging the residual down to the residual on the coarsened grids of this level, in mgRelax.

- NodeMGInterp m mginterp is used for interpolating the correction in mgRelax.

\subsection{The class LevelNodeSolver}

The LevelNodeSolver class solves elliptic equations on a level using the multigrid method. It is used by AMRNodeSolver to solve at the base level.

The API is as follows.

- void define(

$\begin{array}{ll}\text { const DisjointBoxLayout\& } & \text { a_grids, } \\ \text { const DisjointBoxLayout* } & \text { a_gridsCoarsePtr, } \\ \text { const ProblemDomain\& } & \text { a_domain, } \\ \text { Real } & \text { a_dx, } \\ \text { int } & \text { a_refToCoarse, } \\ \text { const NodeMaskLevelOp* const } & \text { a_opin, } \\ \text { int } & \text { a_minLength }=1)\end{array}$

- void define(

$\begin{array}{ll}\text { const DisjointBoxLayout\& } & \text { a_grids, } \\ \text { const DisjointBoxLayout* } & \text { a_gridsCoarsePtr, } \\ \text { const Box\& } & \text { a_domain, } \\ \text { Real } & \text { a_dx, } \\ \text { int } & \text { a_refToCoarse, } \\ \text { const NodeMaskLevelOp* const } & \text { a_opin, } \\ \text { int } & \text { a_minLength }=1)\end{array}$

Sets up the internal state of a LevelNodeSolver object.

Inputs:

- a_grids specifies the layout of the cell-centered grids at this level.

- a_gridsCoarsePtr is a pointer to the layout of the cell-centered grids at the next coarser level, or NULL if there is no coarser level. 
- a_domain specifies the cell-centered problem domain on this level.

- a_dx is the mesh spacing at this level.

- a_refToCoarse is the refinement ratio between this level and the next coarser level. Ignored if there is no coarser level.

- a_opin is a pointer to the NodeMaskLeve10p to use in solving.

- a_minLength is the minimum length of the maximally coarsened box, or 0 if there is to be no coarsening. If a minLength $\geq 1$ then the multigrid solver will coarsen boxes only so far as to maintain the lengths of all boxes as multiples of a_minLength.

- void levelSolve(

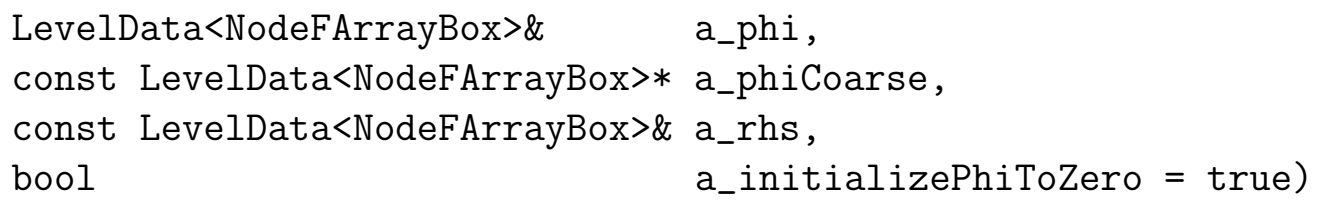

Does a level solve on this level using multigrid and inhomogeneous boundary conditions at coarse/fine interfaces.

Inputs:

- a_phi is initial guess at solution at this level.

- a_phiCoarse is a pointer to solution at next coarser level, or NULL if there is no coarser level.

- a_rhs contains the right-hand side at this level.

- a_initializePhiToZero tells whether to initialize the solution to zero.

\section{Output:}

- a_phi is updated solution at this level.

- void levelSolveH(

$$
\begin{array}{ll}
\text { LevelData }<\text { NodeFArrayBox }>\& & \text { a_phi, } \\
\text { const LevelData }<\text { NodeFArrayBox }>\& & \text { a_rhs, } \\
\text { bool } & \text { a_initializePhiToZero = true) }
\end{array}
$$

Does a level solve on this level using multigrid and homogeneous boundary conditions at coarse/fine interfaces.

Inputs:

- a_phi is initial guess at solution at this level.

- a_rhs contains the right-hand side at this level.

- a_initializePhiToZero tells whether to initialize the solution to zero. 


\section{Output:}

- a_phi is updated solution at this level.

- void setTolerance(Real a_tolerance)

Sets the tolerance in the level solver: iterations end when the norm of the residual is less than a_tolerance times the initial residual. Default is $1.0 e-10$.

- void setOperatorTolerance(Real a_operatorTolerance)

Sets the "operator tolerance" of the solver: iterations end if the ratio of the new residual to the old residual exceeds 1 - a_operatorTolerance and at least m minIter iterations have been performed. Default is $1.0 e-4$.

- void setMaxIter(int a_maxIter)

Sets the maximum number of relaxation iterations. Default is 33 .

- void setMinIter(int a_minIter)

Sets the minimum number of iterations in the solver before it will terminate due to lack of convergence. Default is 4 .

- void setnumSmoothDown(int a_numSmoothDown)

Sets the number of iterations of smoothing for the downward part of the $\mathrm{V}$-cycle in m_levelMG.mgRelax. Default is 4 .

- void setnumSmoothUp (int a_numSmoothUp)

Sets the number of iterations of smoothing for the upward part of the V-cycle in m_levelMG.mgRelax. Default is 4 .

- void setnumBottomGSRB (int a_numBottomGSRB)

Sets the number of iterations of smoothing at the bottom of the V-cycle in m_levelMG.mgRelax. Default is 16 .

- void setBottomSmoothing(bool a_bottomSolveFlag)

Sets whether the smoother is applied at the bottom of the V-cycle in m_levelMG.mgRelax. Default is true.

\section{Principal data members}

- NodeLevelMg m_levelMG is a multigrid level solver object to relax on this level.

- NodeMaskLevelOp* m_levelOpPtr is a pointer to the NodeMaskLevelOp to use in solving.

- LevelData<NodeFArrayBox> m_resid is the residual in the level solve.

- LevelData<NodeFArrayBox> m_corr is the correction in the level solve. 


\subsection{The NodeMaskLeve1Op interface}

NodeMaskLeve10p is a pure base class to encapsulate level operations for node-centered elliptic solvers.

- virtual NodeMaskLevelOp* new_levelop ()$=0$

Returns a pointer to a new instance of NodeMaskLevelOp of the same type. This gets around the "no virtual constructor" rule.

- virtual void define(

const DisjointBoxLayout\& a_grids, const DisjointBoxLayout* a_gridsCoarsePtr, Real

a_dx, int a_refToCoarse, const ProblemDomain\& a_domain, bool a_homogeneousOnly = false, int a_ncomp = 1) $=0$

- virtual void define( const DisjointBoxLayout\& a_grids, const DisjointBoxLayout* a_gridsCoarsePtr, Real int const Box\& a_dx, a_refToCoarse, bool int a_domain, a_homogeneousOnly = false, a_ncomp = 1) =0

Full define function. Makes all coarse/fine information and sets internal variables. Inputs:

- a_grids specifies the layout of the cell-centered grids at this level.

- a_gridsCoarsePtr is a pointer to the layout of the cell-centered grids at the next coarser level, or NULL if there is no coarser level.

- a_domain specifies the cell-centered problem domain on this level.

- $a_{-} d x$ is the mesh spacing at this level.

- a_refToCoarse is the refinement ratio between this level and the next coarser level. Ignored if there is no coarser level.

- a_homogeneous0nly specifies whether all coarse/fine interpolation is homogeneous.

- a_ncomp is the number of components in the operator argument. 
- virtual void define(

const NodeMaskLevelOp* a_opfine, int

a_refToFine) $=0$

Full define function. Makes all coarse/fine information and sets internal variables from finer NodeMaskLevelOp. Any NodeMaskLevelOp defined with this function is not able to execute inhomogeneous boundary conditions at the coarse/fine interface. Inputs:

- a_opfine is the finer-level operator.

- a_refToFine is the refinement ratio between this level and the next finer level.

- virtual void CFInterp(
LevelData $<$ NodeFArrayBox $>\&$
a_phi,
const LevelData<NodeFArrayBox $>\&$
a_phiCoarse, bool
a_inhomogeneous) $=0$

Fills the nodes on the coarse/fine interface with interpolated data from the coarser level.

\section{Inputs:}

- a_phi is the solution on the current level.

- a_phiCoarse is the solution at the next coarser level.

- a_inhomogeneous specifies whether the physical boundary condition is inhomogeneous.

- virtual void homogeneousCFInterp(

LevelData $<$ NodeFArrayBox $>$ \& a_phi) $=0$

Zeroes out the nodes that lie on the interface with the coarser level.

Input:

- a_phi is the solution on this level.

\section{Output:}

- a_phi is the solution on this level with interface nodes set to zero.

- virtual void $\operatorname{smooth}($

LevelData $<$ NodeFArrayBox $>\&$ a_phi, const LevelData<NodeFArrayBox $>\&$ a_rhs $)=0$

Smoother. This smooths the solution (in the multigrid sense) on a level. It assumes that the problem has already been put into residual-correction form, so that coarse/fine boundary conditions are homogeneous.

Inputs: 
- a_phi is the solution on this level.

- a_rhs is the right-hand side on this level.

\section{Output:}

- a_phi is the smoothed solution on this level.

- virtual void applyOpH(

LevelData<NodeFArrayBox $>\&$ a_LofPhi, LevelData $<$ NodeFArrayBox $>\&$ a_phi $)=0$

- virtual void applyOpI(

LevelData $<$ NodeFArrayBox $>$ \& a_LofPhi, LevelData $<$ NodeFArrayBox $>\& \quad$ a_phi, const LevelData<NodeFArrayBox $>*$ a_phiCoarsePtr $)=0$

- virtual void applyOpHcfIphys (

LevelData<NodeFArrayBox $>$ \& a_LofPhi, LevelData $<$ NodeFArrayBox $>\&$ a_phi $)=0$

- virtual void applyOpIcfHphys ( LevelData $<$ NodeFArrayBox $>\&$ LevelData $<$ NodeFArrayBox $>$ \& a_LofPhi, const LevelData<NodeFArrayBox $>*$ a_phiCoarsePtr $)=0$

Evaluate the operator. The four functions differ in whether the boundary conditions are homogeneous or inhomogeneous on the coarse/fine interface and on the physical boundary:

\begin{tabular}{l|cc} 
function & coarse/fine interface & physical boundary \\
\hline applyOpH & homogeneous & homogeneous \\
applyOpI & inhomogeneous & inhomogeneous \\
applyOpHcfIphys & homogeneous & inhomogeneous \\
applyOpIcfHphys & inhomogeneous & homogeneous
\end{tabular}

Inputs:

- a_phi is the solution on this level.

- a_phiCoarsePtr is a pointer to the solution at the next coarser level, required for inhomogeneous coarse/fine boundary conditions.

\section{Output:}

- a_LofPhi is the result of the operator on this level. 
- virtual void residualH(

$\begin{array}{ll}\text { LevelData }<\text { NodeFArrayBox }>\& & \text { a_resid, } \\ \text { LevelData }<\text { NodeFArrayBox }>\& & \text { a_phi, } \\ \text { const LevelData }<\text { NodeFArrayBox }>\& & \text { a_rhs })=0\end{array}$

- virtual void residualI(

LevelData $<$ NodeFArrayBox $>\& \quad$ a_resid,

LevelData $<$ NodeFArrayBox $>\& \quad$ a_phi,

const LevelData<NodeFArrayBox $>*$ a_phiCoarsePtr,

const LevelData $<$ NodeFArrayBox $>\&$ a_rhs $)=0$

- virtual void residualHcfIphys (

$\begin{array}{ll}\text { LevelData }<\text { NodeFArrayBox }>\& & \text { a_resid, } \\ \text { LevelData }<\text { NodeFArrayBox }>\& & \text { a_phi, } \\ \text { const LevelData }<\text { NodeFArrayBox }>\& \text { a_rhs })=0\end{array}$

- virtual void residualIcfHphys (

$\begin{array}{ll}\text { LevelData }<\text { NodeFArrayBox }>\& & \text { a_resid, } \\ \text { LevelData }<\text { NodeFArrayBox }>\& & \text { a_phi, } \\ \text { const LevelData }<\text { NodeFArrayBox }>* & \text { a_phiCoarsePtr, } \\ \text { const LevelData }<\text { NodeFArrayBox }>\& & \text { a_rhs })=0\end{array}$

Calculate the residual of the operator. If $L$ is the operator, $\varphi$ is the solution to which the operator is applied, and $\rho$ is the right-hand side, then the residual is simply $\rho-L(\varphi)$. The four functions differ in whether the boundary conditions are homogeneous or inhomogeneous on the coarse/fine interface and on the physical boundary:

\begin{tabular}{l|cc} 
function & coarse/fine interface & physical boundary \\
\hline residualH & homogeneous & homogeneous \\
residualI & inhomogeneous & inhomogeneous \\
residualHcfIphys & homogeneous & inhomogeneous \\
residualIcfHphys & inhomogeneous & homogeneous
\end{tabular}

Inputs:

- a_phi is the solution on this level.

- a_phiCoarsePtr is a pointer to the solution at the next coarser level, required for inhomogeneous coarse/fine boundary conditions.

- a_rhs is the right-hand side on this level.

\section{Output:}

- a_resid is the residual on this level. 
- virtual void bottomSmoother (

$$
\begin{array}{ll}
\text { LevelData }<\text { NodeFArrayBox }>\& \quad \text { a_phi, } \\
\text { const LevelData }<\text { NodeFArrayBox }>\& \text { a_rhs })=0
\end{array}
$$

Performs smoothing at the bottom level. This is used when it is not possible to coarsen the grid. Typically, the function is either a point relaxation or a conjugategradient type of method.

Inputs:

- a_phi is the solution on this level.

- a_rhs is the right-hand side on this level.

\section{Output:}

- a_phi is the smoothed solution on this level.

- virtual void levelPreconditioner(

$$
\begin{array}{ll}
\text { LevelData }<\text { NodeFArrayBox }>\& & \text { a_phihat, } \\
\text { const LevelData }<\text { NodeFArrayBox }>\& & \text { a_rhshat })=0
\end{array}
$$

Applies preconditioner. In the notation of $\left[\mathrm{BBC}^{+} 94\right]$, if the preconditioner is $M$, which is an approximation to the operator, then this solves the related equation $M \hat{\phi}=\hat{\rho}$. In the AMR multigrid implementation, this function is generally most often used by the bottom solver.

\section{Input:}

- a_rhshat is the right-hand side of the preconditioning.

\section{Output:}

- a_phihat is the result of the preconditioning.

\subsubsection{The NodeMaskBaseBottomSmoother interface}

NodeMaskBaseBottomSmoother is a pure base class to encapsulate operations for smoothers used by node-centered elliptic solvers.

- virtual NodeMaskBaseBottomSmoother* new_bottomSmoother $($ ) const $=0$

Returns a pointer to a new instance of NodeMaskBaseBottomSmoother of the same type.

- virtual void doBottomSmooth(

$$
\begin{array}{ll}
\text { LevelData }<\text { NodeFArrayBox }>\& & \text { a_phi, } \\
\text { const LevelData<NodeFArrayBox }>\& & \text { a_rhs, } \\
\text { const BoxLayoutData< BaseFab<int }>>\& & \text { a_mask, } \\
\text { NodeMaskLevelOp* } & \text { a_levelop_ptr })=0
\end{array}
$$


Performs smoothing of $L(\varphi)=\rho$.

Inputs:

- a_phi is the solution on a level.

- a_rhs is the right-hand side on a level.

- a_mask is the mask array from REACHABLENODES on this level.

- a_levelop_ptr is a pointer to an instance of the operator.

\section{Output:}

- a_phi is the smoothed solution on the level.

Examples of smoothers are conjugate gradient and BiCGStab, described in [BBC $\left.{ }^{+} 94\right]$. These are implemented as the NodeMaskCGSmoother and NodeMaskBiCGStabSmoother classes, respectively, described in detail in [McC02].

\subsubsection{The class NodeMaskPoissonOp}

NodeMaskPoissonOp is a subclass of NodeMaskLevelOp that includes operations for the discrete Laplacian operator. Here we describe only the functions and members of NodeMaskPoissonOp that are not described in the section on NodeMaskLevelOp.

- void setBottomSmoother (const NodeMaskBaseBottomSmoother\& a_bottomSmoother)

Sets the bottom smoother to be used in the smooth function. The default bottom smoother for NodeMaskPoissonOp is an object of class NodeMaskCGSmoother.

- void setInterpolationDegree(int a_interpolationDegree)

Sets the degree of interpolation to be used in NodeQuadCFInterp: 1 for linear in two dimensions or bilinear in three dimensions; 2 for quadratic in two dimensions or biquadratic in three dimensions. Default is 2 . This function must be called before define. The interpolation degree cannot be changed later without calling define again.

- void setDomainNodeBC(const DomainNodeBC\& a_dombcIn)

Sets the boundary conditions of the physical domain.

\subsection{The class AMRNodeSolverAlt}

The AMRNodeSolverAlt class solves an elliptic equation on an adaptive mesh refinement hierarchy using an alternative algorithm to the AMR multigrid solver implemented in AMRNodeSolver. AMRNodeSolverAlt solves one level at a time, from coarsest to finest, interpolating from coarser to finer solutions as it proceeds. See Figure 4.2 for a chart of major classes used in AMRNodeSolverAlt. 


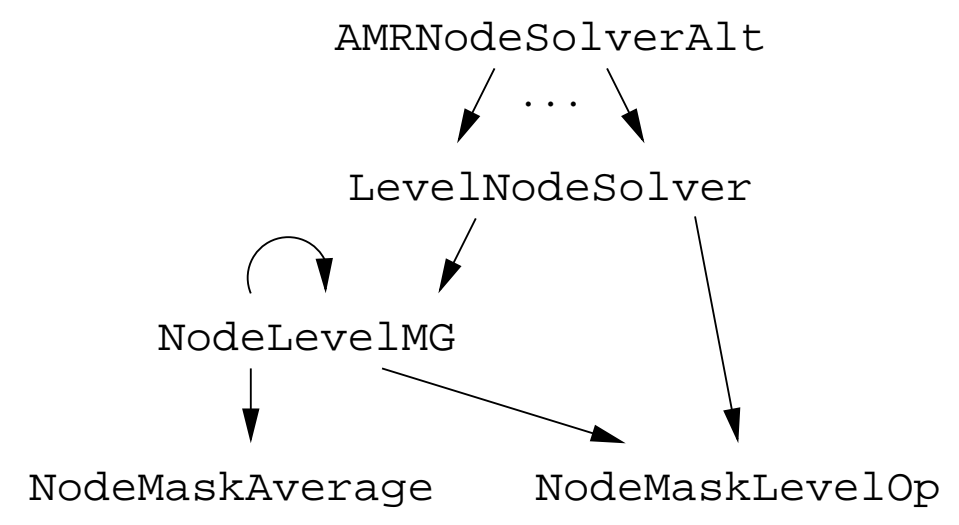

Figure 4.2: This chart shows the major classes of AMRNodeElliptic used by AMRNodeSolverAlt. An arrow from one class to another indicates that an instance of the first class defines an instance of the second class. In each case, only one instance of the second class is defined, except that the AMRNodeSolverAlt defines an instance of LevelNodeSolver for each level in the AMR hierarchy.

- void define(

$\begin{array}{ll}\text { const Vector<DisjointBoxLayout>\& } & \text { a_gridsLevel, } \\ \text { const Vector<ProblemDomain>\& } & \text { a_domainLevel, } \\ \text { const Vector<Real>\& } & \text { a_dxLevel, } \\ \text { const Vector<int>\& } & \text { a_refRatio, } \\ \text { int } & \text { a_numLevels, } \\ \text { int } & \text { a_lBase, } \\ \text { const NodeMaskLevelOp* const } & \text { a_opin, } \\ \text { int } & \text { a_minLength }=1)\end{array}$

- void define(

$\begin{array}{ll}\text { const Vector<DisjointBoxLayout }>\& & \text { a_gridsLevel, } \\ \text { const Vector }<\text { Box }>\& & \text { a_domainLevel, } \\ \text { const Vector }<\text { Real }>\& & \text { a_dxLevel, } \\ \text { const Vector<int>\& } & \text { a_refRatio, } \\ \text { int } & \text { a_numLevels, } \\ \text { int } & \text { a_lBase, } \\ \text { const NodeMaskLevelOp* const } & \text { a_opin, } \\ \text { int } & \text { a_minLength }=1)\end{array}$

Sets up the internal state of an AMRNodeSolverAlt object with information about the grid hierarchy and the operator. The arguments are the same as for the define function of AMRNodeSolver.

- void solveAMR(

$$
\text { Vector }<\text { LevelData }<\text { NodeFArrayBox }>*>\& \quad \text { a_phiLevel, }
$$


const Vector<LevelData<NodeFArrayBox $>*>$ \& a_rhsLevel)

Solves the elliptic equation over a hierarchy of levels. The arguments are the same as for the solveAMR function of AMRNodeSolver.

- void setTolerance(Real a_tolerance)

Sets the tolerance in the one-level solvers: iterations at a level end when the norm of the residual is less than a_tolerance times the initial residual. Default is $1.0 e-10$.

- void setMaxIter(int a_maxIter)

sets the maximum number of iterations in the solvers at all levels. Default is set in LevelNodeSolver.

- void setBottomSmoothing(bool a_doBottomSmooth)

sets whether the smoother is applied at the bottom of the $\mathrm{V}$-cycle in the function NodeLevelMG: :mgRelax. Default is true.

\section{Principal data members.}

- Vector<LevelNodeSolver*> $\mathrm{m}_{-}$levelSolver is a vector of length $\mathrm{m} \_$numLevels containing pointers to LevelNodeSolver objects, each of which manages the data and operations on one level.

- Vector<DisjointBoxLayout> m_gridsLevel, Vector<Box>m_domainLevel, Vector<Real> m_dxLevel, Vector<int> m_refRatio, int m_numLevels, int $\mathrm{m}_{-}$lBase are set to the arguments a_gridsLevel, a_domainLevel, a_dxLevel, a_refRatio, a_numLevels, a_lBase in the constructor. 


\section{Chapter 5}

\section{$\mathrm{C}++$ Classes for Two-Level Operators}

\subsection{The class NodeMaskAverage}

The NodeMaskAverage class is used for replacing coarse-level data with an average of fine-level data in the classes NodeLevelMG and AMRNodeLevelMG.

- void define(

const DisjointBoxLayout\& a_gridsFine, const DisjointBoxLayout\& a_gridsCoarse, int a_numcomps, int a_refRatio, const ProblemDomain\& a_domainFine, Real a_dx)

- void define(

const DisjointBoxLayout\& a_gridsFine, const DisjointBoxLayout\& a_gridsCoarse, int

a_numcomps,

int

a_refRatio,

const Box\&

a_domainfine, Real

a_dx)

Defines the internal state of the NodeMaskAverage object.

Inputs:

- a_gridsFine specifies the layout of the cell-centered grids at the finer level.

- a_gridsCoarse specifies the layout of the cell-centered grids at the coarser level.

- a_numcomps is the number of components in the data.

- a_refRatio is the refinement ratio between the two levels.

- a_domainfine specifies the cell-centered problem domain 
- $a_{-} d x$ is the mesh spacing at the finer level.

- void define(

const DisjointBoxLayout\& a_gridsCoarse, int a_numcomps,

int a_refRatio,

const ProblemDomain\& a_domainFine,

Real a_dx)

- void define(

const DisjointBoxLayout\& a_gridsCoarse,

int a_numcomps,

int a_refRatio,

const Box\& a_domainFine,

Real a_dx)

Defines the internal state of the NodeMaskAverage object. The arguments are the same as that of the longer define function above, except that a_gridsFine is not present; the grids at the finer level are taken to be refinements of the grids at the coarser level.

- void averageToCoarse(

LevelData $<$ NodeFArrayBox $>\&$ a_coarse, LevelData<NodeFArrayBox $>$ \& a_fine)

Replaces coarse-level data with an average of nearby finer-level cells' data, on the interior of the coarsened finer-level domain. Elsewhere, the coarse-level data is unchanged.

Input:

- a_fine is finer-level data.

\section{Output:}

- a_coarse is coarser-level data with interior nodes replaced by average of finerlevel data.

\subsection{The class NodeMGInterp}

The NodeMGInterp class is used for interpolating data from a coarser to a finer AMR level in the class AMRNodeLevelMG.

- void define(

const DisjointBoxLayout\& a_grids, int

a_numcomps , 
int

const ProblemDomain\&

Real
a_refRatio,

a_domain,

$a_{-} d x$ )

- void define(

const DisjointBoxLayout\& a_grids,

int

int

a_numcomps,

const Box\&

a_refRatio,

Real

a_domain,

$\left.a_{-} d x\right)$

Defines the internal state of the NodeMGInterp object.

Inputs:

- a_grids specifies the layout of the cell-centered grids at the finer level.

- a_numcomps is the number of components in the data.

- a_refRatio is the refinement ratio between the two levels.

- a_domain specifies the cell-centered problem domain on the finer level.

$-a_{-} d x$ is the mesh spacing at the finer level.

- void interpToFine(

LevelData $<$ NodeFArrayBox $>\& \quad$ a_fine, const LevelData<NodeFArrayBox $>\&$ a_coarse)

bool

a_sameGrids $=$ false)

Modifies the finer-level data by adding interpolated values of the coarser-level data. Input:

- a_fine is original finer-level data.

- a_coarse is coarser-level data.

- a_sameGrids specifies whether the finer-level grids are refinements of the coarser-level grids.

\section{Output:}

- a_fine is updated finer-level data.

\subsection{The class NodeQuadCFInterp}

The NodeQuadCFInterp class interpolates data from a coarse level to a fine level on coarse/fine interfaces. 
- void define(

$\begin{array}{ll}\text { const DisjointBoxLayout\& } & \text { a_grids, } \\ \text { Real } & \text { a_dx, } \\ \text { const ProblemDomain\& } & \text { a_domain, } \\ \text { const LayoutData<NodeCFIVS }>* \text { const } & \text { a_loCFIVS, } \\ \text { const LayoutData<NodeCFIVS }>* \text { const } & \text { a_hiCFIVS, } \\ \text { int } & \text { a_refToCoarse, } \\ \text { int } & \text { a_interpolationDegree }=2, \\ \text { int } & \text { a_ncomp }=1)\end{array}$

- void define(

$\begin{array}{ll}\text { const DisjointBoxLayout\& } & \text { a_grids, } \\ \text { Real } & \text { a_dx, } \\ \text { const Box\& } & \text { a_domain, } \\ \text { const LayoutData<NodeCFIVS }>* & \text { const } \\ \text { const LayoutData<NodeCFIVS }>* \text { const } & \text { a_hiCFIVS, } \\ \text { int } & \text { a_refToCoarse, } \\ \text { int } & \text { a_interpolationDegree }=2, \\ \text { int } & \text { a_ncomp }=1)\end{array}$

Defines the internal state of the NodeQuadCFInterp object.

Inputs:

- a_grids specifies the layout of the cell-centered grids at the finer level.

- $a_{-} d x$ is the mesh spacing at the finer level.

- a_domain specifies the cell-centered problem domain on the finer level.

- a_loCFIVS and a_hiCFIVS are pointers to arrays of length SpaceDim of LayoutData<NodeCFIVS>, each of which holds information about nodes on the coarse/fine interface for each finer-level box. The underlying layout in every case is a_grids. The arrays of LayoutData<NodeCFIVS $>$ are indexed by dimension, with a_loCFIVS for faces on the low sides and a hiCFIVS for faces on the high sides.

- a_refToCoarse is the refinement ratio between the two levels. It must be a power of 2 .

- a_interpolationDegree is the degree of interpolation. It is 1 if interpolation is linear in two dimensions or bilinear in three dimensions. It is 2 if interpolation is quadratic in two dimensions or biquadratic in three dimensions.

- a_ncomp is the number of components in the data.

- void coarseFineInterp(

LevelData<NodeFArrayBox $>\& \quad$ a_phiFine, const LevelData<NodeFArrayBox>\& a_phiCoarse, 
bool

a_inhomogeneous)

Fills the nodes of the fine level of the coarse/fine interfaces with interpolated data from the coarser level.

Inputs:

- a_phiCoarse is coarser-level data.

- a_inhomogeneous specifies whether the physical boundary condition is inhomogeneous.

\section{Output:}

- a phiFine is the finer-level data, modified at nodes on interfaces with the coarser level.

- void setDomainNodeBC (const DomainNodeBC\& a_dombcIn)

Sets the boundary conditions of the physical domain. This is required if the refinement ratio is more than 2 , because then the boundary conditions need to be set on the intermediate levels between successive interpolations by refinement ratio of 2 .

\subsection{The class NodeCFIVS}

The NodeCFIVS class determines the set of nodes that lie on the coarse/fine interface, for a particular face of a particular box at the finer level.

- void define(

$\begin{array}{ll}\text { const ProblemDomain\& } & \text { a_domain, } \\ \text { const Box\& } & \text { a_box, } \\ \text { const DisjointBoxLayout\& } & \text { a_levelBoxes, } \\ \text { int } & \text { a_idir, } \\ \text { Side: : LoHiSide } & \text { a_hiorlo) }\end{array}$

- void define(

$\begin{array}{ll}\text { const Box\& } & \text { a_domain, } \\ \text { const Box\& } & \text { a_box, } \\ \text { const DisjointBoxLayout\& } & \text { a_levelBoxes, } \\ \text { int } & \text { a_idir, } \\ \text { Side: :LoHiSide } & \text { a_hiorlo) }\end{array}$

Defines the internal state of the NodeCFIVS object.

Inputs:

- a_domain specifies the cell-centered problem domain on the finer level.

- a_box specifies the finer-level box. 
- a_levelBoxes specifies the layout of all cell-centered boxes at the finer level.

- a_idir and a_hilorlo specify the particular face of a_box.

- const IntVectSet\& getFineIVS() const

Returns the indices of the finer-level nodes on this face that also lie on the interface with the coarser level. The IntVectSet excludes indices of nodes that lie on the boundary of the problem domain, and nodes that are on faces of any other boxes in a_levelBoxes on the side of a_box specified by a_idir and a_hilorlo. See Figure 5.1.
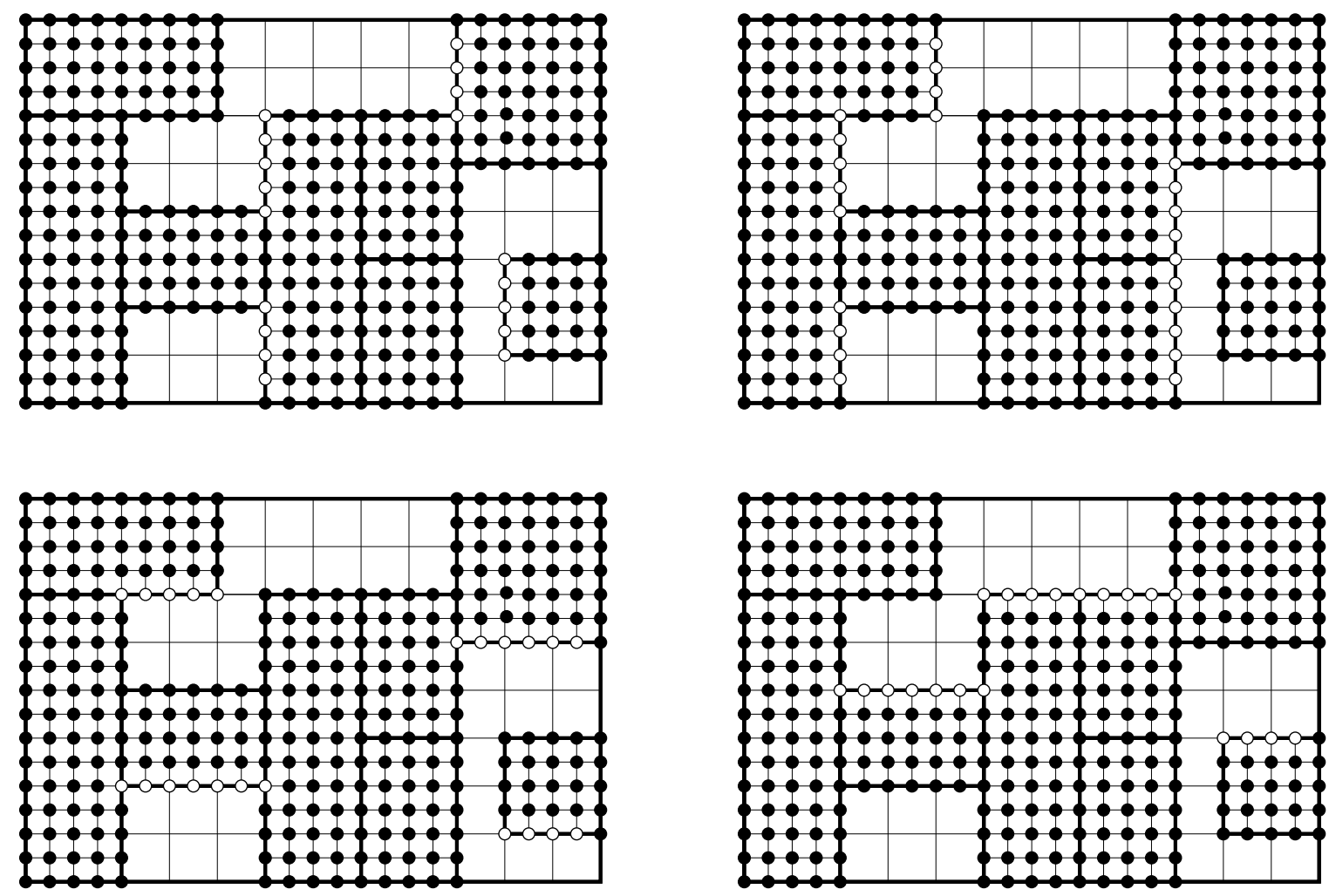

Figure 5.1: This figure indicates, with hollow circles, all nodes with indices returned by getFineIVS over all settings of a_box in a two-dimensional layout over a non-periodic domain.

Top: a_idir $=0$, and a_hiorlo = (left) Side: $:$ Lo or (right) Side: $:$ Hi.

Bottom: a_idir $=1$, and a hiorlo $=$ (left) Side: $:$ Lo or (right) Side: :Hi. 


\section{Chapter 6}

\section{External Boundary Conditions}

Boundary conditions on the faces of the rectangular problem domain are enforced with the class DomainNodeBC, which contains a pointer to an instance of the class FaceNodeBC for each boundary face. The user adds a FaceNodeBC-derived class for each face to enforce particular boundary conditions.

The AMRNodeElliptic package allows boundary conditions that are expressed as

$$
A \frac{\partial \varphi}{\partial n}+B \varphi=C
$$

for each node lying on the boundary, where $A, B$, and $C$ may vary over the boundary. The class derived from FaceNodeBC provides a function to fill $A, B$, and $C$ on a boundary face. DomainNodeBC calls this function and fills the boundary nodes appropriately. Examples:

- Dirichlet: $A=0, B=1$. For homogeneous, $C=0$.

- Neumann: $A=1, B=0$. For homogeneous, $C=0$.

The user needs to define an instance of DomainNodeBC only once, regardless of the number of levels of refinement. The problem domain and the mesh spacing for the level are inputs to the functions that set and apply the boundary conditions.

\subsection{The DomainNodeBC class}

There is one instance of DomainNodeBC for the whole problem domain.

- DomainNodeBC()

This constructor defines the object in an unusable state until the user calls setFaceNodeBC for each face.

- void setFaceNodeBC(

const FaceNodeBC\& a_bc) 
Sets boundary conditions at a face.

Input:

- a_bc is boundary condition at a face.

- void applyHomogeneousBCs (

$\begin{array}{ll}\text { NodeFArrayBox\& } & \text { a_state, } \\ \text { const ProblemDomain\& } & a_{-} \text {domain, } \\ \text { Real } & \left.a_{-} d x\right) \text { const }\end{array}$

- void applyHomogeneousBCs (

NodeFArrayBox\& a_state, const Box\& a_domain, Real a_dx) const

- void applyInhomogeneousBCs (

NodeFArrayBox\& a_state,

const ProblemDomain\& a_domain,

Real a_dx) const

- void applyInhomogeneousBCs (

NodeFArrayBox\& a_state,

const Box\& a_domain,

Real a_dx) const

Apply boundary conditions. The difference between applyHomogeneousBCs and applyInhomogeneousBCs is that applyHomogeneousBCs sets $C=0$ in (6.1).

Inputs:

- a_domain is the cell-centered problem domain on the level.

- $a_{-} d x$ is the mesh spacing on the level.

\section{Output:}

- a_state contains the data. Data at nodes on the boundary of the problem domain are modified according to the boundary conditions. Data at other nodes are unchanged.

\subsection{The FaceNodeBC interface}

There is a FaceNodeBC object for each of the $2 *$ SpaceDim faces of the problem domain.

- FaceNodeBC(
int
a_dir,
Side: :LoHiSide a_sd) 
- FaceNodeBC(

$$
\begin{aligned}
& \text { int a_dir, } \\
& \text { Side: :LoHiSide a_sd, } \\
& \text { const Interval\& a_comps) }
\end{aligned}
$$

Constructor that defines boundary conditions on a face.

Inputs:

- a_dir is dimension of face, 0 up to SpaceDim-1.

- a_sd is either Side: :Lo or Side: :Hi in specifying the face.

- a_comps, if present, specifies the interval over which components of the data will have boundary values set. If absent, then the interval is $(0: 0)$.

- virtual FaceNodeBC $*$ new_boxBC () const $=0$

Returns a pointer to a new instance of FaceNodeBC of the same type.

- void define(

$$
\text { int } \text { Side: :LoHiSide a_dir, }
$$

- void define(

$$
\begin{array}{ll}
\text { int } & \text { a_dir, } \\
\text { Side: : LoHiSide } & \text { a_sd, } \\
\text { const Interval\& } & \text { a_comps) }
\end{array}
$$

Define the FaceNodeBC object with the same arguments as the constructors.

- virtual void applyHomogeneousBCs (
FArrayBox\&
a_state,

const ProblemDomain\& a_domain,

Real

$a_{-} d x$ ) const

- virtual void applyHomogeneousBCs (

FArrayBox\& a_state,

const Box\& a_domain,

Real a_dx) const

- virtual void applyInhomogeneousBCs (
FArrayBox\&
a_state,

const ProblemDomain\& a_domain, Real

$a_{-} d x$ ) const

- virtual void applyInhomogeneousBCs (

FArrayBox\& a_state, const Box\& a_domain, 


$$
\text { Real a_dx) const }
$$

The difference between applyHomogeneousBCs and applyInhomogeneousBCs is that applyHomogeneousBCs sets $C=0$ in (6.1).

\section{Inputs:}

- a_domain is the cell-centered problem domain on the level.

- $a_{-} d x$ is the mesh spacing on the level.

\section{Output:}

- a_state contains the data. Data on nodes of a_state that lie on the boundary of the cell-centered problem domain a_domain are modified according to the boundary conditions. Data on other nodes of a_state are unchanged.

- virtual void fillBCValues(

$$
\begin{array}{ll}
\text { FArrayBox\& } & \text { a_neumfac, } \\
\text { FArrayBox\& } & \text { a_dircfac, } \\
\text { FArrayBox\& } & \text { a_inhmval, } \\
\text { Real } & \text { a_dx, } \\
\text { const ProblemDomain\& } & \text { a_domain) const }=0
\end{array}
$$

- virtual void fillBCValues(

FArrayBox\& a_neumfac,

FArrayBox\& a_dircfac,

FArrayBox\& a_inhmval,

Real a_dx,

const Box\& a_domain) const $=0$

Sets the coefficients in (6.1). This function must be provided in the derived class. Inputs:

- a_dx is the mesh spacing on the level.

- a_domain is the cell-centered problem domain on the level.

Outputs: Data in these node-centered FArrayBoxes are set on the nodes that lie on this face of the boundary of a_domain. When called by applyEitherBCs, all three of them have the same underlying node-centered box, which will be a subbox of this boundary face. In the boundary condition equation $A \frac{\partial \varphi}{\partial n}+B \varphi=C$ (6.1):

- a_neumfac contains $A$.

- a_dircfac contains $B$.

- a_inhmval contains $C$. 


\section{Bibliography}

$\left[\mathrm{BBC}^{+} 94\right]$ R. Barrett, M. Berry, T. Chan, J. Demmel, J. Donato, J. Dongarra, V. Eijkhout, R. Pozo, C. Romine, and H. Van der Vorst. Templates for the Solution of Linear Systems: Building Blocks for Iterative Methods. SIAM, Philadelphia, PA, 1994.

[CGL $\left.{ }^{+} 00\right]$ P. Colella, D. T. Graves, T. J. Ligocki, D. F. Martin, D. Modiano, D. B. Serafini, and B. Van Straalen. Chombo Software Package for AMR Applications - Design Document. unpublished, 2000.

[McC02] P. McCorquodale. AMRNodeElliptic Software Package: Node-Centered AMR for Elliptic Problems. unpublished, 2002. 